\title{
Auf die Größe kommt es an! Neue empirische Evidenz zur Wahlbeteiligung in Gemeinden
}

\author{
Reinhard Heinisch • Armin Mühlböck (C)
}

Online publiziert: 7. Oktober 2016

(C) Der/die Autor(en) 2016. Dieser Artikel ist eine Open-Access-Publikation.

Zusammenfassung Studien zu „Size and Democracy“ argumentieren, dass für kleinere politische Einheiten eine höhere Wahlbeteiligung zu erwarten ist. Empirische Tests belegen diese Annahme in weiten Teilen. Neuere, komplexere Analysen, die Individual- und Kontextdaten integrieren, kommen aber für die Schweiz, Norwegen, Dänemark und die Niederlande zu nicht eindeutigen Ergebnissen. Um den Zusammenhang neu zu bewerten, greift die vorliegende gepoolte Zeitreihenanalyse auf sieben Kommunalwahlen in allen Gemeinden des österreichischen Bundeslandes Salzburgs (ohne die Stadt Salzburg) zwischen 1979 und 2009 zurück. Die vorliegende Mehrebenenanalyse (nicht-hierarchisches cross-classified Modell), welches Kontexteffekte berücksichtigt, liefert neue Evidenz: Die Größe der Gemeinde beeinflusst die Wahlbeteiligung in Gemeinden in hohem Ausmaß negativ. Dabei kann vor allem die Wechselwirkung mit dem Grad gesellschaftlicher Fraktionalisierung als ausschlaggebend angesehen werden.

Schlüsselwörter Gemeinden · Wahlen · Wahlbeteiligung · Wahlkreise · „Size and Democracy“

\section{Size matters - New empirical evidence on voter turnout in municipalities}

Abstract Studies on "size and democracy" argue that smaller political units should exhibit higher voter turnout rates. Empirical tests largely confirm this assumption. In contrast, more complex analyses that integrate individual and contextual data arrive at inconclusive results with regard to Switzerland, Norway, Denmark and the

Prof. R. Heinisch · A. Mühlböck ( $ه)$

Department of Political Science, University of Salzburg, Rudolfskai 42, 5020 Salzburg, Österreich

E-Mail: Armin.Muehlboeck@sbg.ac.at

Prof. R. Heinisch

E-Mail: Reinhard.C.Heinisch@sbg.ac.at 
Netherlands. In order to reevaluate the relationship between municipality size and turnout, this article uses a pooled time series analysis to study seven local elections in all municipalities of the Austrian state of Salzburg (excluding the city of Salzburg) between 1979 and 2009. The multi-level analysis (non-hierarchical cross-classified model) that accounts for context effects provides new evidence that the size of the municipality has a negative impact on turnout rates. Above all, the interaction between the size of the municipalities and the degree of societal fractionalization appears to be a decisive factor.

Keywords Municipalities · Elections · Turnout · Constituencies · "Size and democracy"

\section{Einleitung}

Die Gemeinden werden oft als „Schulen der Demokratie“ und als „Legitimationszentren“ für die übergeordneten politischen Ebenen beschrieben. Darauf aufbauend wird den lokalen Einheiten eine wichtige Funktion bei der Förderung der allgemeinen demokratischen Entwicklung zugesprochen (Vetter 2002, S. 16; Roth 2009). In Gemeinden sind die Herausforderungen an politische Gestaltung oft weniger komplex, die Barrieren für Beteiligung niedriger und die Entscheidungsträger aufgrund der geringen Distanz zu den Wählern und Wählerinnen besser und direkter ansprechbar. Geht es um die Beteiligung bei Gemeindewahlen sind allerdings große Unterschiede zwischen Gemeinden beobachtbar, die in einem ansonsten relativ homogenen regionalen Umfeld liegen. Es gibt ein Nebeneinander von Gemeinden mit reger Beteiligung und solchen mit viel niedrigeren Partizipationsraten. Im österreichischen Bundesland Salzburg, dem Gegenstand unserer Untersuchung, liegt die Wahlbeteiligung bei Gemeindewahlen zwischen rund $70 \%$ und fast $100 \%$. Was erklärt diese Variation der Partizipationsraten bei Gemeindewahlen?

Studien unter dem Titel „Size and Democracy“ argumentieren, dass für kleinere politische Einheiten eine höhere Wahlbeteiligung zu erwarten ist. Vielfach durchgeführte empirische Tests der „Gemeindegröße“ zeigen auch mit hoher Wahrscheinlichkeit, dass die Einwohnerzahl einer Gemeinde die Variation der Wahlbeteiligung signifikant beeinflusst. Ladner (2009) regte allerdings an, diesen Zusammenhang zu hinterfragen, weil bei neueren Messungen in der Schweiz, in Norwegen, in Dänemark und in den Niederlanden der postulierte negative Effekt der Größe der Einheit auf die Partizipationsrate nicht nachgewiesen werden konnte. Der wissenschaftliche Output zum Effekt der "Size“ hat auch durch die Analyse demokratiepolitischer Effekte von Gemeindestrukturreformen neuen Schwung bekommen (Hansen 2013; Heinisch et al. 2016; Hicks 2006; Horiuchi et al. 2015; Shimizu 2012), zumal diese vorrangig die Größenstruktur der kommunalen Ebene betreffen und die Chance liefern, im Setting eines natürlichen Experiments Hypothesen zu testen.

Soziologische und politikwissenschaftliche Forschung (siehe z. B. Coffe und Geys 2006; Geys 2006) führte zudem vor Augen, dass (zunehmende) gesellschaftliche Heterogenität negativ mit der Partizipationsrate bei Wahlen korreliert. Wir werden dazu 
argumentieren, dass kleinere Gemeinden den negativen Effekt gesellschaftlicher Heterogenität mindern können.

Vor diesem Hintergrund werden wir die Wirkung des Faktors „Population Size“ auf die Partizipationsraten bei Gemeindewahlen einem neuerlichen Test unterziehen und mit weiteren sozio-ökonomischen Determinanten sowie politischen und institutionellen Einflussgrößen kontrollieren. Wir greifen dazu auf Daten zu sieben Kommunalwahlen in den Gemeinden im Bundesland Salzburg (ohne die Stadt Salzburg) zwischen 1979 und 2009 zurück (insgesamt 826 Wahlgänge). Zum Einsatz kommt ein nicht-hierarchisches Cross-Classified-Modell. Fallauswahl und Modellsetting erlauben einen hohen Grad an interner Validität unserer Untersuchung, weil viele politisch-institutionelle Merkmale auf Partizipationsraten, wie das Wahlsystem, die Gleichzeitigkeit von z. B. First- und Second-Order-Elections (Concurrent Elections) oder eine bestimmte Tradition im Wahlverhalten (Previous Turnout), als kontrolliert betrachtet werden können.

\section{Wahlbeteiligung in der Demokratietheorie}

Wenden wir uns der Erklärung der Varianz der Wahlbeteiligung zu, so liefert ein einleitender Blick auf die demokratietheoretische Diskussion zur Relevanz der Partizipationsrate hilfreiche Hintergrundinformationen zum Gegenstand der Analyse. Dabei stehen die Fragen im Vordergrund, inwieweit eine hohe Wahlbeteiligung als Funktionsvoraussetzung für Demokratien angesehen werden kann oder eine sinkende Wahlbeteiligung ein bedeutsames demokratiepolitisches Problem darstellt (siehe z. B. Lijphart 1997; Verba 2003). Die Antworten fallen ambivalent aus und sind auf im Hintergrund wirkende normative Denkmuster zurückzuführen (Schmidt 2000, S. $451 \mathrm{ff}$ ). Eher partizipatorisch ausgerichtete Ansätze wie jener der deliberativen Demokratie (Habermas), das Modell der starken Demokratie (Barber), der expansiven (Warren) oder assoziativen Demokratie (Hirst) stellen in puncto politischer Beteiligung höhere Ansprüche als die traditionell-liberale oder realistische Demokratietheorie, die das Kriterium der Repräsentation und deren Legitimation in den Vordergrund rücken (Marxer 2004, S. 9).

Dabei gilt bei den erstgenannten Ansätzen die Wahl einmal als Startpunkt eines in alle gesellschaftlichen Bereiche hineinwirkenden Beteiligungsprozesses, während bei letzteren neben dem Wahlakt, keine weiteren partizipativen Anforderungen gestellt werden. So kann Wählen nur als wichtig erachtet werden, um auf diese Art und Weise den Elitenwettbewerb auf bestimmte Zeit zu entscheiden und Macht zuzuweisen. Eine hohe Wahlbeteiligung hat dabei zunächst keine besondere Funktion und sinkende Partizipationsraten können sogar als vorteilhaft interpretiert, da ,,[...] die Einmischung in das Regieren durch die Regierten gering bleibt“" (Kornelius und Roth 2004, S. 23). Eine hohe Wahlbeteiligung würde vielmehr auf Konflikte in der Gesellschaft hindeuten, während ,[...] eine niedrige Wahlbeteiligung [...] ein Ausdruck der generellen Zufriedenheit der Bürger mit dem demokratischen System und seiner Politik“ (Merkel und Petring 2011, S. 8) wäre. Eine niedrige oder sinkende Wahlbeteiligung gilt allerdings nur so lange als unproblematisch, wie 
[...] alle sozialen Schichten und Bevölkerungsgruppen gleichermaßen wenig zur Wahl gehen [...]. Wäre es hingegen so, dass nur noch bestimmte Bevölkerungsgruppen von ihrem Wahlrecht Gebrauch machen, dann bestünde die Gefahr, dass die politische Gleichheit als fundamentales Prinzip der Demokratie einer Ungleichheit der Interessenartikulation weicht (Merkel und Petring 2011, S. 8).

Somit impliziert auch die Argumentation in der restriktiven Demokratietheorie ein bestimmtes kritisches Ausmaß an Wahlbeteiligung. Merkel und Petring erklären mit Verweis auf empirische Wahl- und Demokratiestudien von Schäfer 2010, Kohler 2006, Lijphart (1997), Dalton (1996) und Topf (1995), ,[...] dass mit sinkender Wahlbeteiligung die soziale Selektivität zunimmt“ (Merkel und Petring 2011, S. 8 f.), weil benachteiligte gesellschaftliche Gruppen eher und stärker als andere Fraktionen auf ihr Wahlrecht verzichten.

Nach dieser Hinführung auf der Grundlage normativer Aussagen, lässt sich der Schluss ziehen, dass der Wahlbeteiligung, selbst wenn diese aus unterschiedlichen demokratietheoretischen Ansätzen betrachtet wird, eine nicht unwesentliche Rolle beigemessen wird. Darauf aufbauend wenden wir uns dem empirisch-analytischen Schwerpunkt der Arbeit und zunächst der theoretischen Argumentation zu.

\section{Zentrales Argument}

Vetter (2008) und Freitag (1996, S. 4) verweisen auf Studien unter dem Titel „Size and Democracy" (Dahl und Tufte 1973; Verba et al. 1979; Blais und Dobrzynska 1998) und argumentieren, dass für kleinere politische Einheiten (Faktor „Population Size") eine höhere Wahlbeteiligung zu erwarten ist. Diese Variation der Partizipationsrate bei regionalen und kommunalen Wahlen konnte auch in Großbritannien (Newton 1982, S. 199), generell Westeuropa und den USA nachgewiesen werden (Morlan 1984, S. 469), wenngleich die Stärke des Effektes unterschiedlich ausfiel. Empirische Evidenz für den negativen Effekt der Größe politischer Einheiten auf die Wahlbeteiligung gibt es auch für die Schweiz (Ladner 2002), für Dänemark (Mouritzen 1989), Spanien (Justel 1995), die Niederlande (Denters et al. 2014) und Kanada (Kushner et al. 1997).

Das Argument für diese negative Beziehung wird vor allem aus Ansätzen der Rational-Choice-Schule abgeleitet, wonach der ,rationale Wähler“ vor allem nutzenund ergebnisorientiert handelt. Dabei geht es aber nicht unmittelbar um individuelle Vorteilserwartungen, die sich durch die Wahl der einen oder anderen wahlwerbenden Gruppe ergeben. Wichtig ist vielmehr die Wahrnehmung, ob die einzelne Stimme einen Unterschied in puncto Wahlausgang bewirkt. Dieser Eindruck wird unter anderem beeinflusst von der Anzahl der Wahlberechtigten. Der Nutzeneffekt, der aus einer Wahlbeteiligung gezogen werden kann, ist in kleineren Gemeinden tendenziell größer, weil die eigene Stimme in Relation zu allen anderen Wahlberechtigten mehr Einfluss hat und damit entscheidender ist (Geys 2006, S. 642). Zudem wirkt sich in kleineren Gemeinden die geringere Distanz und damit direktere Beziehung zwischen Politiker und Politikerinnen auf der einen sowie Bürgern und Bürgerinnen auf 
der anderen Seite günstig auf die Partizipationsraten aus. In kleineren Gemeinden sind die Wege kürzer und der Ressourceneinsatz bei der Beteiligung am politischen Diskurs ist tendenziell geringer als in größeren Einheiten (Vetter 2002, S. 16). Die Motive der Gemeindepolitik und der lokalen Parteien können in kleineren Gemeinden einfacher und unmittelbarer vermittelt werden, oft sogar im Face-to-FaceKontakt. Nicht zu vergessen ist die leichtere Überschaubarkeit des unmittelbaren Lebensumfeldes (Anderwald 2008, S. 24). Auch dem Aspekt der ,,sozialen Kontrolle" ist Aufmerksamkeit zu widmen. In der klassischen wahlsoziologischen Debatte wird dieser Theoriestrang auch unter dem Titel „Population Concentration“ diskutiert und fokussiert dabei Disparitäten zwischen urbanen und ruralen Räumen. In eher städtisch strukturierten lokalen Einheiten, die geprägt sind durch gesellschaftliche Individualisierung und einem hohen Grad an Anonymität, soll der soziale Druck auf die Ausübung des individuellen Wahlrechts weit weniger stark ausgeprägt sein, wie in eher ländlich geprägten Gebieten (Vetter 2008, S. 63 f.; Geys 2006, S. 643 f.). Die dahingehenden Testergebnisse zeigen allerdings ein uneinheitliches Bild. Geys (2006, S. 643) zieht in seiner dahingehenden Besprechung den Schluss, ,that the relation between population concentration and voter turnout is weak". Ein Grund könnte darin zu finden sein, dass - die lokale Ebene im Blick - auch der ländliche Raum in regionale Zentren und Peripherien und damit wiederum in urbaner und ruraler strukturierte lokale Einheiten zerfällt. Das führt zur Überlegung, dass der Theoriestrang der „Population Concentration“ in das Argument zur „Population Size“ integriert werden sollte. Auszugehen ist ja davon, dass „soziale Kontrolle“ unabhängig von der Lage der Gemeinde - in kleineren Einheiten stärker zum Tragen kommt als in größeren und auch vor diesem Hintergrund für kleinere politische Einheiten eine höhere Partizipationsrate beobachtbar sein sollte (Anderwald 2008, S. 24).

Ladner (2009, S. 6) regte allerdings erst kürzlich an, diesen Zusammenhang zu hinterfragen, weil ,new and rather complex analyses using individual and contextual data for Switzerland, Norway, Denmark and the Netherlands [...] do not find a consistent negative effect of size on turnout". Die Messungen könnten aber dadurch beeinflusst sein, dass gerade die skandinavischen Länder und auch die Niederlande eine durchschnittliche Gemeindegröße von 30.000 und mehr Einwohner und Einwohnerinnen aufweisen (Italien ca. 7000, Deutschland ca. 6000, Österreich ca. 3400, Frankreich ca. 1600). Für die Schweiz ist bei der Interpretation der Wahlbeteiligung die Bedeutung der direkten Demokratie mit zu beachten.

Strukturreformen auf kommunaler Ebene setzen vor allem bei der Veränderung der Größe der lokalen Einheiten an. Durch die Fusion kleinerer Gemeinden oder einer kleinen Gemeinde mit einer größeren, steigt die Anzahl der Wahlberechtigten im letzteren Fall für die Einwohnerinnen und Einwohner der kleinen Gemeinde sprunghaft an, während sich die Anzahl der Mandate, wenn überhaupt, dann nur wenig ändert. Somit verringern sich in Folge einer Reform das relative Gewicht einer Wählerstimme und damit die Chance, eine Wahl zu beeinflussen. Dieser Logik folgend, sollte in Reformgemeinden die Wahlbeteiligung sinken bzw. stärker zurückgehen als in Gemeinden, die von strukturellen Maßnahmen nicht betroffen sind. Neuere Arbeiten im Kontext kommunaler Strukturreformen, die mittels einer experimentellen Anordnung den Effekt der „Gemeindegröße“ auf die Wahlbeteili- 
gung testen, bieten allerdings keine klare Linie über Richtung, Stärke oder generell die Relevanz des Einflusses. So zeigte Hicks (2006), dass sich, in Folge der Fusion von Gemeinden zur Großstadt Toronto, die Wahlbeteiligung sogar erhöhte. In anderen Fällen wiederum blieb der Reformeffekt gänzlich insignifikant (Bhatti und Hansen 2010), während weitere Analysen Evidenz für einen Rückgang der Partizipationsrate bei Kommunalwahlen (Heinisch et al. 2016; Morlan 1984) lieferten.

Auch Blais und Dobrzynska (1998, S. 242) wiesen unter Bezugnahme auf Dahl und Tufte (1973) darauf hin, dass, ,the relationship between community size and turnout is far from being unambiguous." Allerdings halten es Blais und Dobrzynska letztlich mit Verba, Nie \& Kim (Verba et al. 1979), die einen höheren Grad an politischer Partizipation in kleineren Einheiten festgestellt haben. Vor allem Geys (2006, S. 642) führt in seiner Besprechung über die Ergebnisse von insgesamt 83 empirischen Studien zur Wahlbeteiligung dann klar vor Augen, dass, auch wenn einzelne Anomalien beobachtet wurden, die vielfach durchgeführten Tests der ,Gemeindegröße" letztlich mit hoher Wahrscheinlichkeit darauf schließen lassen, dass „population size has a statistically significant negative effect on turnout“. Für die vorliegende Untersuchung kann daher wie folgt erwartet werden:

H1 Je größer die Gemeinde, desto niedriger ist die Wahlbeteiligung bei Gemeindewahlen.

\section{Kontrollvariablen}

\subsection{Gesellschaftliche Heterogenität - Population Heterogeneity}

Zahlreiche Studien fokussieren den Effekt der persönlichen Ressourcenausstattung und die Wirkung des sozialen Umfelds auf das individuelle politische Engagement (Kaniovski und Mueller 2006, S. 405 f.; mit Verweis auf Aldrich 1997; Lindert 1996; La Ferrara 2002; Miguel und Gerty 2005). Forschungsarbeiten zeigten, dass das Interesse an Politik (auf allen Ebenen) und die Beteiligung an Wahlen positiv mit Bildung und Einkommen korrelieren. Die Kollektivebene betrachtend, nimmt das politische Engagement in Gemeinden aber mit zunehmender gesellschaftlicher Heterogenität tendenziell ab, gleich ob ethnische Aspekte, das Einkommen oder die Bildung gemessen werden. Die Politikwissenschaft (Rallings et al. 1994) zeigte, dass ein hoher Grad an lokaler Diversität den politischen Ausgleich zwischen den gesellschaftlichen Gruppen erschwert und die Effektivität des lokalen politischen Systems („Political Efficacy“) darunter leidet (Kaniovski und Mueller 2006, S. 406). Die darauf aufbauende Forschung führte vor Augen (Geys 2006, S. 644 ff.), dass eher homogene Strukturen höhere Partizipationsraten hervorbringen, weil der soziale Zusammenhalt dort aufgrund gemeinsamer Interessen tendenziell stärker ausgeprägt ist. Geht es um den Effekt der „Population Heterogeneity“ auf die Wahlbeteiligung, zeichnet die politikwissenschaftliche Theorie allerdings kein einheitliches Bild. So werden ebenso plausibel positive Effekte gesellschaftlicher Heterogenität auf die Beteiligung bei Gemeindewahlen deshalb angenommen (Zimmer 1976, S. 689 f.), weil in ausdifferenzierten lokalen Gesellschaften entstehende Interessenskonkurrenz 
bei Inhalten, Kandidaten und politischen Gruppierungen den lokalen politischen Wettbewerb nicht hemmen, sondern vielmehr stimulieren und sich sodann positiv auf die Wahlbeteiligung auswirken kann (Geys 2006, S. 644 f.; Kaniovski und Mueller 2006, S. 406 f.; Zimmer 1976, S. 689 f.).

Verglichen mit anderen sozio-ökonomischen Determinanten der Wählerpartizipation wird der Faktor „Population Heterogeneity“ (ausführlich Geys 2006, S. 644 ff.) in Wahlbeteiligungsstudien allerdings selten gemessen. Wie die theoretischen Argumente schon vermuten lassen, liefert der Test-output zudem widersprüchliche Ergebnisse. Geys (2006, S. 645) resümiert, dass hinsichtlich der Assoziation zwischen „Population Homogeneity“ und Wahlbeteiligung: ,the estimated average effect [...] is never significantly different from 0, though it is mostly negative “. Hinsichtlich des Aspekts „Population Heterogeneity“ kann daher wie folgt erwartet werden:

H2 Je heterogener die lokale Gesellschaft in sozio-ökonomischer Hinsicht strukturiert ist, desto niedriger die Wahlbeteiligung bei Gemeindewahlen.

\subsection{Interaktion - Population Size und Population Heterogeneity}

Wie noch im Detail zu beschreiben sein wird, erfolgt hier die Beobachtung der lokalen Variation sozio-ökonomischer Heterogenität mit dem kommunalen „Tertiärisierungsgrad“. Der Begriff ,Tertiärisierung“ erfasst wichtige Aspekte des sozioökonomischen Strukturwandels, der sich, beginnend in den 1970er-Jahren, auf unterschiedliche Art und Weise und zum Teil zeitversetzt, vor allem in den westlichen Industriestaaten vollzogen hat. „Tertiärisierung“ bedeutet dabei nicht nur, dass der dritte Wirtschaftssektor gegenüber dem ersten (Land- und Forstwirtschaft, Fischerei und Bergbau) und vor allem zweiten Sektor (produzierendes Gewerbe) hinsichtlich der Anzahl der Beschäftigten immer mehr an Bedeutung gewinnt. „Tertiärisierung“ schließt auch die Dynamik mit ein, dass in puncto Berufsstruktur eine Tendenz zu höher- und hochqualifizierten Tätigkeiten und intellektueller Arbeit verbunden mit längeren Schul- und Ausbildungszeiten besteht (Baethge 2000, S. 87; Elkmann et al. 1994). Wir argumentieren, dass „Tertiärisierung“ hinsichtlich der Bildungs-, Berufs- und damit auch Einkommensstrukturen in einem zunehmend höheren Grad an sozialer Diversität resultiert.

Die Entwicklungen verlaufen in den Gemeinden allerdings höchst unterschiedlich, wobei sowohl die Lage und als auch die Gemeindegröße eine wichtige Rolle spielen. Sozi-ökonomischer Wandel wirkt vor allem in regionalen und überregionalen Arbeitsmarktzentren und deren Umland in einem hohen Grad gesellschaftlicher Fraktionalisierung. Dort ist allein schon aufgrund des Angebots an postsekundären und tertiären Bildungseinrichtungen und deren leichterer Erreichbarkeit eine viel stärke Bildungsdynamik beobachtbar als in peripher gelegenen Gemeinden. Zudem bieten diese Räume für jüngere Menschen im höheren Bildungssegment ob der Jobchancen im (höheren) Dienstleistungsbereich einen attraktiven Lebensraum. Regionale und überregionale Arbeitsmarktzentren und deren Umland sind deshalb auch einer verstärkten Zuwanderung aus Landgemeinden ausgesetzt, die diese Erwerbschancen nicht bieten können (Schipfer 2005, S. 16; Mühlböck 2010; Mühlböck und Dirninger 2010; Mühlböck 2013, S. 237). Die soziologische Forschung zeigte 
dazu, dass hinsichtlich des sozialen Lebens in der Gemeinde dem Aspekt der Stabilität der lokalen Bevölkerung eine wichtige Rolle zukommt (Coffe und Geys 2006, S. 1067). Soziales Kapital entwickelt sich in Gemeinden, die - unabhängig von der Größe - einer hohen Zuwanderung ausgesetzt sind, nur erschwert. Dem sozialen Milieu in kleineren Gemeinden wird dabei ein moderierender positiver Effekt zugeschrieben, weil sich in der Bevölkerung aufgrund der Überschaubarkeit gegenseitiges Vertrauen und sozialer Zusammenhalt - und wohl auch sozialer Druck - leichter und schneller entwickeln können. Für zugewanderte und damit ortsfremde Personen erhöhen sich aber jedenfalls die Kosten der lokalen politischen Beteiligung, weil Politik, Gesellschaft und lokale Problemlagen weitgehend unbekannt sind (Geys 2006, S. 644 f.). Wanderungsbewegungen finden nicht nur zwischen Stadt und Land statt. Geht es um die eher kleineren Gemeinden im unmittelbaren Umfeld einer Stadt, spielen die Prozesse der Suburbanisierung eine wichtige Rolle. Vor allem jüngere Familien aus dem Bildungsbürgertum wandern aus den unterschiedlichsten Gründen, wohl aber vor allem auf der Suche nach einem familientauglicheren Lebensumfeld, aus der Kernstadt in das Umland ab. Die betroffenen Stadtumlandgemeinden (Suburbs) oder Teile davon entwickeln sich dabei oft zu sogenannten „Schlafdörfern“ oder „Bedroom Communities“. Die Zugewanderten wohnen bzw. „schlafen“ zwar in der Wohnsitzgemeinde, ,leben“ aber in der nächstgelegenen Stadt, die in puncto Beruf, Ausbildung, Freizeit und Kultur den Lebensmittelpunkt darstellt. „Bedroom communities“ sind üblicherweise „characterized by a lack of citizen involvement “ (Shinn 1999, S. 25 f.). Den Blick auf die eben argumentierte Wechselwirkung zwischen der „Gemeindegröße“ und den Effekten lokaler gesellschaftlicher Fraktionalisierung gerichtet, ist wie folgt zu ergänzen:

H3 Mit der lokalen Bevölkerungszahl verstärkt sich der negative Effekt gesellschaftlicher Heterogenität auf die Wahlbeteiligung.

\subsection{Finanzkraft - Economic Efficiency}

Freitag (1996, S. 15 ff.) argumentiert, dass das Ausmaß der Partizipation bei Wahlen auch von der wirtschaftlichen Leistungskraft und Entwicklung eines gesellschaftlichen Systems abhängt und sich dabei ein hoher Grad an sozio-ökonomischer Modernisierung positiv auf die Wahlbeteiligung auswirken sollte (,Economic Efficiency“). Finanzkräftige lokale Einheiten sollten auf eine positive Infrastrukturentwicklung hinsichtlich der Schulen, der Vereinbarkeit von Familie und Beruf oder in puncto Freizeiteinrichtungen und im Kulturbereich verweisen können. Diese lokalen politischen Systeme sollten einen höheren Legitimitätsgrad erreichen und auf diese Art und Weise - angelehnt an die Erkenntnisse von Powell (Powell 1982, S. 37; Blais und Dobrzynska 1998, S. 242) - die Beteiligung bei Gemeindewahlen positiv beeinflussen. Folgt man allerdings demokratietheoretischen Überlegungen, wonach eine sinkende oder niedrige Wahlbeteiligung auch als Ausdruck von Stabilität und Vertrauen in die demokratischen Institutionen und ihrer Problemlösungskompetenz interpretiert werden kann, könnte mit dem Ausmaß der materiellen Ressourcenausstattung einer Gemeinde die Wahlbeteiligung auch sinken. Den folgenden Analysen 
soll in Anlehnung an die klassischen wahlsoziologischen Theorien aber folgende Annahme zu Grunde liegen:

H4 Je finanzkräftiger die Gemeinde, desto höher fällt die Wahlbeteiligung bei Gemeindewahlen aus.

\subsection{Wettbewerb im Parteiensystem - Political Competition}

Allgemeiner Stand der Erkenntnis ist, dass ein ausgeprägter Parteienwettbewerb (Political Competition, auch „Closeness“ oder im Umkehrschluss „Marginality“) (Geys 2006, S. 646 f.) die Höhe der Wahlbeteiligung positiv beeinflusst (Blais und Dobrzynska 1998, S. 249; Vetter 2008, S. 60). Das dahinter liegende Argument wird als „Downsian Closness Hypothesis“ geführt und bringt zum Ausdruck, dass mit der Enge eines Rennens der individuell wahrnehmbare Effekt - oder die Relevanz - einer Wähler- bzw. Wählerinnenstimme steigt. Die höhere Nutzenerwartung, die dann mit einer Wahlbeteiligung verbunden ist, wirkt sich positiv auf die Partizipationsrate aus (Geys 2006, S. 647). Die damit verbundene Erwartung ist wie folgt formuliert:

H5 Je ausgeprägter der lokale Parteienwettbewerb, desto höher ist die Wahlbeteiligung.

\subsection{Fraktionalisierungsgrad im Parteiensystem - Political Fragmentation}

Neben dem Wettbewerbsgrad wird auch der Anzahl der zur Wahl antretenden Parteien und damit dem Fragmentierungsgrad im lokalen Parteiensystem Bedeutung zugewiesen (Political Fragmentation). Je mehr Parteien zur Wahl antreten, desto mehr Auswahlmöglichkeiten stehen zur Verfügung und desto eher ist die Möglichkeit gegeben, dass ein den persönlichen Interessen nahekommendes Angebot vorhanden ist. Dies wiederum wirkt mobilisierend und erzeugt positive Effekte auf die Wahlbeteiligung (Freitag 1996, S. 10). In der Literatur sind allerdings, ausgehend von Grundannahmen der Rational-Choice-Theorie, auch Hinweise darauf zu finden, dass ein zu breit gefächertes Angebot auch dazu führen kann, dass der Auswahlprozess einen zu hohen persönlichen Ressourceneinsatz erfordert und gleichzeitig der Einfluss der Wähler und Wählerinnen auf die Machtzuteilung sinkt (Freitag 1996, S. 10). Diese Abläufe können sich negativ auf die Partizipationsrate auswirken (Blais und Dobrzynska 1998, S. 242; Vetter 2008, S. 60). Geys (2006, S. 649 f.) erklärt, dass die durchgeführten Tests zur Wirkung der politischen Fragmentierung kein einheitliches Bild ergeben. Vetter (2008, S. 61), Freitag (1996, S. 7) und Ladner (2009, S. 11) argumentierten aber positive Effekte wie folgt:

H6 Je ausgeprägter die politisch Fragmentierung im lokalen Parteiensystem, desto höher ist die Wahlbeteiligung bei Gemeindewahlen.

Geht es um die Effekte weiterer politisch-institutioneller Merkmale auf Partizipationsraten bei Wahlen, werden unter anderem das Wahlsystem, die Gleichzeitigkeit von z. B. First- und Second-Order-Elections (Concurrent Elections) oder einer bestimmten Tradition im Wahlverhalten (Previous Turnout) diskutiert (Vetter 2008, 
S. 62 ff.; Geys 2006, S. 646 ff.; Freitag 1996). ${ }^{1}$ Wie einleitend erwähnt, müssen diese Faktoren nicht extra gemessen werden, sondern können einerseits aufgrund der Fallauswahl (Gemeindewahlen im Bundesland Salzburg) und andererseits aufgrund des noch zu besprechenden Modellsetting (Mehrebenenanalyse - Cross Classified Modell) als kontrolliert betrachtet werden.

\section{Operationalisierung}

\subsection{Gemeindegröße - Population Size}

Die Messgrundlage für die „Gemeindegröße“ ist die Anzahl der Einwohner und Einwohnerinnen einer Gemeinde jeweils zum 1.1. im betreffenden Wahljahr. Die Wahlbeteiligung ergibt sich aus dem Anteil der Wählenden an den Wahlberechtigten in einer Gemeinde (Geys 2006, S. 638 f).

\subsection{Gesellschaftliche Heterogenität - Population Heterogeneity}

Für die Messung des Grades sozio-ökonomischer gesellschaftlicher Diversität auf der Aggregatebene wird üblicher Weise das Durchschnittseinkommen erhoben (Geys 2006, S. 645). Daneben erfolgten Datensammlungen auch über die Anteile der Beschäftigten in den drei großen Wirtschaftssektoren (Dolezal und Poier 2012, S. 16). Hier wird die lokale Variation sozio-ökonomischer Heterogenität mit dem kommunalen „Tertiärisierungsgrad“ erfasst. So kann der Nachteil der geringen Variation auf der Ebene der Gemeinden in der traditionellen Messung mit dem Durchschnittseinkommen vermieden werden. Zudem finden dann die hier angepeilten Prozesse eines sozio-ökonomischen Wandels, der gesellschaftliche Diversität nach sich zieht, in die Untersuchung Eingang. Die Messung erfolgt mit einem Index, der sich aus zwei Komponenten zusammensetzt: dem Anteil der unselbstständig Beschäftigen im höheren Dienstleistungsbereich (ohne Handel, Verkehr und Tourismus = Higher Services Sector - HSS) an der Gesamtanzahl der unselbstständig Beschäftigen am Wohnort und dem Anteil der Maturanten und Maturantinnen sowie Akademiker und Akademikerinnen an der Gesamtbevölkerung über 15 Jahren (Higher Education $\mathrm{HE})$ in der jeweiligen Gemeinde:

$$
T I=\frac{H S S * H E}{100}
$$

\footnotetext{
1 Eine zusammenfassende Darstellung der in empirischen Studien am häufigsten eingesetzten Faktoren liefert Geys (2006). Eine dahingehend kompakte und dennoch breit gefächerte Darstellung möglicher Makrofaktoren liefert z. B. Freitag (1996). Der Autor differenziert dabei institutionelle, sozio-politische und politisch-kulturelle (wie Konfessionszugehörigkeit, Organisationsgrad der Parteien und Gewerkschaften), sozioökonomische und demographische Faktoren (wie Bildungsniveau, Alter und Frauenanteil) und schließlich eine historische Dimension von Partizipation. Eine gebündelte Darstellung findet sich auch bei Vetter (2008).
} 


\subsection{Interaktion - Population Size und Population Heterogeneity}

Die Argumente führten darauf hin, dass die Variation der lokalen sozio-ökonomischen Heterogenität von der Lage, von Wanderungsbewegungen und der Gemeindegröße beeinflusst wird. Mit dem Indikator zur Messung lokaler gesellschaftlicher Fraktionalisierung, dem lokalen Tertiärisierungsgrad, können sowohl die Lage der Gemeinde als auch Wandungsbewegungen erfasst werden, weil vor allem die Gemeinden, die selbst Arbeitsmarktzentren sind oder in deren Umland liegen, einen hohen Tertiärisierungsgrad und damit ein hohes sozio-ökonomisches Heterogenitätsniveau aufweisen. Zudem verzeichnen Gemeinden, für die ein vergleichsweise hohes Tertiärisierungsniveau festzuhalten ist, auch gleichzeitig ein relativ starkes Bevölkerungswachstum. Diese Bevölkerungszunahme ist vor allem auf Zuwanderung zurückzuführen (Filipp und Kurz 2014, S. 31 f.).

Die Messung der „Population Heterogeneity“ umfasst allerdings nicht den Effekt der Gemeindegröße, weil es sowohl kleine wie auch große Gemeinden mit niedrigem oder hohem sozio-ökonomischen Fraktionalisierungsgrad gibt. Der nicht additive Interaktionseffekt zwischen „Population Size“ und „Population Heterogeneity“ wird als Produktterm der betroffenen Variablen modelliert.

\subsection{Finanzkraft - Economic Efficiency}

Für die Messung der materiellen lokalen Ressourcen einer Gemeinde wird der amtliche Finanzkrakftindikator für lokale Einheiten verwendet, der wie folgt ermittelt wird: „Summe der Grundsteuer, Kommunalsteuer, Gewerbesteuer (nach dem Gewerbeertrag und dem Gewerbekapital), Lohnsummensteuer und Getränkesteuer und der den Gemeinden zugekommenen Ertragsanteile an den gemeinschaftlichen Bundesabgaben ohne Spielbankabgabe“.2 Um einen Effekt der Finanzkraft auf lokale Partizipationsraten festhalten zu können, wird die durchschnittliche Finanzkraft einer Gemeinde in den letzten fünf Jahren vor der jeweiligen Gemeindewahl als Bezugsgröße definiert.

\subsection{Wettbewerb im Parteiensystem - Political Competition}

Die Standardmessgröße der „Political Competition“ ist, ausgehend vom Wahlergebnis, der Abstand zwischen dem Anteil der ersten und der zweiten Partei.

\subsection{Fraktionalisierungsgrad im Parteiensystem - Political Fragmentation}

Für die Messung des Fragmentierungsgrades wird neuerlich eine Standardmessgröße, der Laakso-Taagepera-Index (i. e., effektive Anzahl der Parteien) eingesetzt (Feld et al. 2007, S. 101).

\footnotetext{
2 Siehe dazu: http://www.ris.bka.gv.at abgerufen am 14.3.2016.
} 


\section{Daten}

Die oben stehende Darstellung (Abb. 1) zeigt die Entwicklung der durchschnittlichen Partizipationsraten in Salzburger Gemeinden beginnend mit den Gemeindewahlen im Jahr 1979. ${ }^{3}$ Deutlich wird, dass die Wahlbeteiligung ab Mitte der 1980er-Jahren leicht zu sinken begann, und zwar von im Schnitt $86 \%$ im Jahr 1984 auf $81 \%$ im Jahr 2009. Es wurden aber nie Werte unter $80 \%$ erreicht. Die Karte (Abb. 2) unterstreicht den Eindruck, dass die Partizipationsraten in den Salzburger recht unterschiedlich ausfielen.

Bei den betrachteten sieben Wahlgängen in 118 Gemeinden im Bundesland Salzburg schwankte die Wahlbeteiligung im Zeitablauf zwischen min. 68,5\% und max. 97,6\% (siehe auch Abb. 1 oben). Die Spannweite innerhalb der beobachteten Abweichungen beträgt somit fast 30 Punkte. Bei drei Viertel der Wahlgänge wurde eine Wahlbeteiligung von rund $80 \%$ und mehr erreicht.

Stellt man die durchschnittliche Wahlbeteiligung in den Salzburger Gemeinden bei Gemeindewahlen jener bei Nationalratswahlen gegenüber, zeigt sich, dass zu Beginn der 1980er-Jahre die Beteiligung bei Nationalratswahlen mit rund $90 \%$ die höchsten Werte annahm (siehe Abb. 1). Diese sank dann aber ab und pendelte sich ab Mitte der 1990er-Jahre bei unter 80 bis rund $75 \%$ ein. Ausnahmen bildeten die

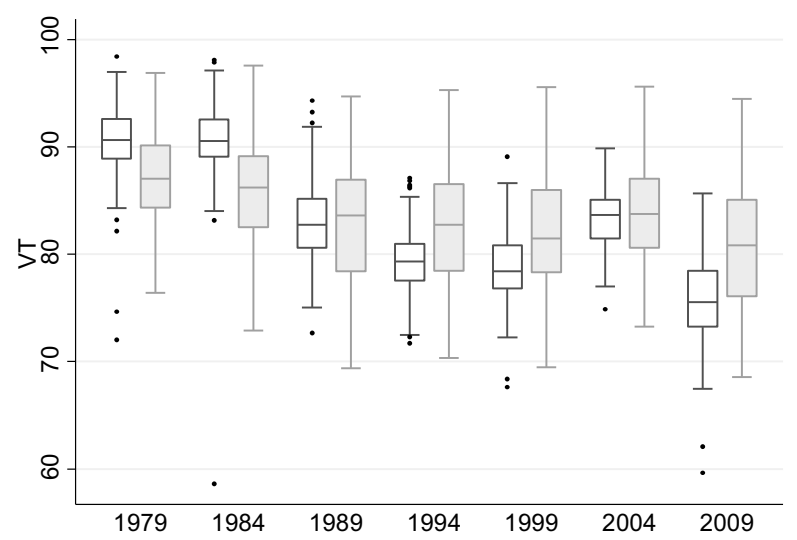

Abb. 1 Wahlbeteiligung bei Gemeinde- (grau) und Nationalratswahlen (weiß) im Bundesland Salzburg (Quelle: Landesstatistik Salzburg und Bundesministerium für Inneres, eigene Berechnung und Darstellung auf Grundlage der Ergebnisse der Gemeindevertretungswahlen in den Salzburger Landgemeinden vom 14.10.1979, 14.10.1984, 08.10.1989, 06.11.1994, 07.03.1999, 07.03.2004, 01.03.2009, der Ergebnisse der Landtagswahlen im Bundesland Salzburg vom 25.03.1979, 25.03.1984, 12.03.1989, 13.03.1994, 07.03.1999, 07.03.2004, 01.03.2009, der Ergebnisse der Nationalratswahlen im Bundesland Salzburg vom 06.05.1979, 24.04.1983, 23.11.1986, 07.10.1990, 09.10.1994, 17.12.1995, 03.10.1999, 24.11.2002, $01.10 .2006,28.09 .2008)$

\footnotetext{
${ }^{3}$ Die durchschnittliche Wahlbeteiligung aller Gemeinden im Bundesland Salzburg spiegelt die Verhältnisse besser wider als ein zusammengefasster Wert, der sich aus allen Wahlberechtigten zu Kommunalwahlen im Bundeland Salzburg im Verhältnis zu allen WählerInnen bei Kommunalwahlen im Bundesland Salzburg ergibt. Siehe dazu z. B.: Landesstatistik Salzburg: www.salzburg.gv.at/20003stat/wahlen/gvw/index. htm.
} 


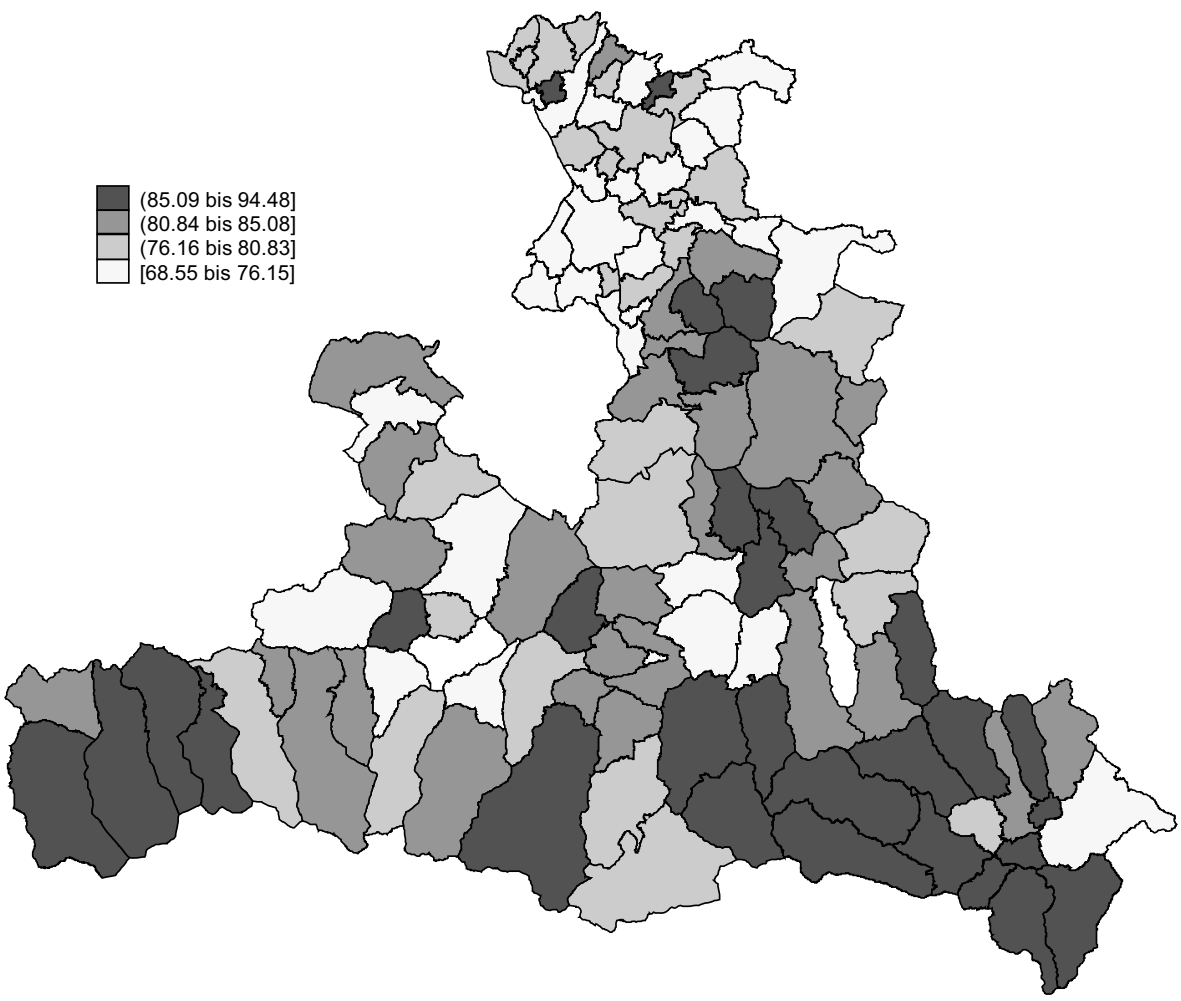

Abb. 2 Wahlbeteiligung im Jahr 2009 in Salzburger Gemeinden (Eigene Darstellung - Quellen: Landesstatistik Salzburg und Bundesministerium für Inneres, eigene Berechnung und Darstellung auf Grundlage der Ergebnisse der Gemeindevertretungswahlen in den Salzburger Landgemeinden vom 14.10.1979, 14.10.1984, 08.10.1989, 06.11.1994, 07.03.1999, 07.03.2004, 01.03.2009, der Ergebnisse der Landtagswahlen im Bundesland Salzburg vom 25.03.1979, 25.03.1984, 12.03.1989, 13.03.1994, 07.03.1999, 07.03.2004, 01.03.2009, der Ergebnisse der Nationalratswahlen im Bundesland Salzburg vom 06.05.1979, 24.04.1983, 23.11.1986, 07.10.1990, 09.10.1994, 17.12.1995, 03.10.1999, 24.11.2002, 01.10.2006, 28.09.2008)

Wahlgänge in den Jahren 1995 und 2002 mit jeweils rund $85 \%$ Wahlbeteiligung. ${ }^{4}$ In der direkten Gegenüberstellung mit den Wahlen auf Bundesebene erweist sich die Wahlbeteiligung bei Kommunalwahlen auf hohem Niveau stabil (Steininger 2006, S. 995). ${ }^{5}$

\footnotetext{
4 Besondere Vorgänge auf Bundesebene haben dabei offenbar zu einer verstärkten Mobilisierung der Wählerinnen und Wähler geführt (1995: Koalitionsbruch im Herbst aufgrund einer Budgetdebatte zwischen SPÖ/Vranitzky und ÖVP/Schüssel und die folgende Dezemberwahl, 2002: Vorgänge rund um einen auBerordentlichen Parteitag der FPÖ im steirischen Knittelfeld im Jahr 2002 haben zu einem Machtwechsel innerhalb der Partei und zum Rücktritt mehrerer FPÖ-Minister geführt. In weiterer Folge ist es zum Bruch der ersten FPÖ/ÖVP-Koalition unter Bundeskanzler Schüssel und schließlich vorgezogenen Neuwahlen gekommen.).

5 In den letzten Jahren ist es auf der lokalen Ebene zu einer Erweiterung der Wahlberechtigten gekommen: zum einen durch das aktive und passive Kommunalwahlrecht der EU-Bürgerinnen und -Bürger und zum anderen durch die Senkung des Wahlalters auf 16 Jahre (i. e., aktive Wahlberechtigung); im Bundesland Salzburg bei Landtags- und Gemeindewahlen seit 2009.
} 
Abb. 3 Anzahl der Gemeinden mit relativ niedriger $(\leq 75 \%$, a) und mit relativ hoher $(\geq 90 \%$, b) Wahlbeteiligung (Quelle: Landesstatistik Salzburg, eigene Berechnung und Darstellung auf Grundlage der Ergebnisse der Gemeindevertretungswahlen in den Salzburger Landgemeinden vom 14.10.1979, 14.10.1984, 08.10.1989, 06.11.1994, 07.03.1999, 07.03.2004, 01.03.2009)

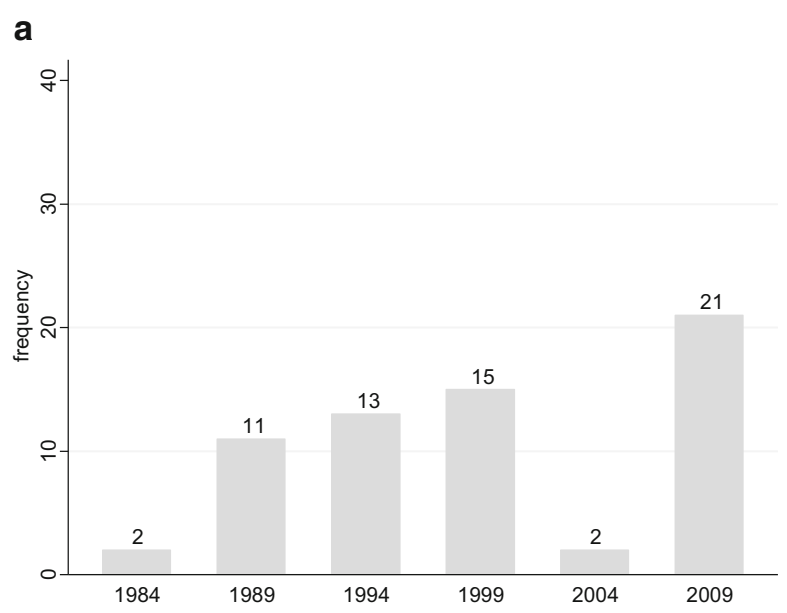

b

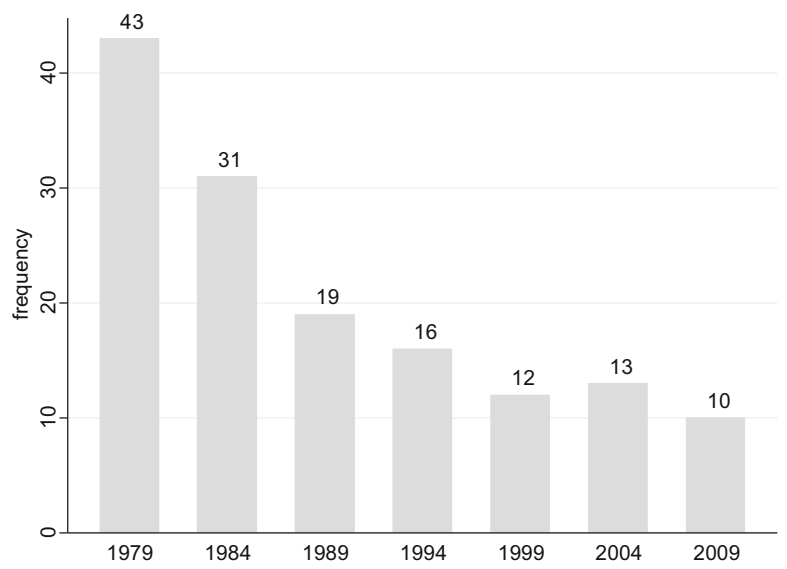

Richtet sich der Blick auf die einzelnen lokalen Einheiten, wird klar, dass sich zwischen 1979 und 2009 die Anzahl der Kommunen mit einer sehr niedrigen Wahlbeteiligung von $75 \%$ oder weniger (gemessen am 10. Percentil), deutlich erhöhte und das vor allem ab Ende der 1980er-Jahre (siehe Abb. 3). Im Wahljahr 1979 gab es noch in keiner Gemeinde eine Wahlbeteiligung von unter 75\%. Im Jahr 1984 machten nur in zwei Gemeinden drei Viertel oder weniger der Wahlberichtigten von ihrem Stimmrecht Gebrauch. Bei der folgenden Gemeindewahl im Jahr 1989 fielen allerdings schon 11 von 118 Gemeinden in diese Kategorie und in den Wahljahren 1994 und 1999 stieg die Anzahl der Gemeinden mit 75\% Wahlbeteiligung oder weniger auf 15 an. Die Kommunalwahlen im Jahr 2004 nahmen insofern eine Sonderstellung ein, als die Wahlbeteiligung gegen den Trend einmalig wieder anstieg. ${ }^{6}$

\footnotetext{
${ }^{6}$ Dieser ,Ausreißer“ ergibt sich aufgrund offenbar besonders mobilisierender Vorgänge rund um die Landtagswahl im Jahr 2004 im Bundesland Salzburg, die zu einem Machtwechsel von ÖVP hin zur SPÖ geführt haben.
} 
Tab. 1 Kriterium, Test- und Kontrollvariablen (eigene Darstellung)

\begin{tabular}{lllllll}
\hline & $\begin{array}{l}\text { Wahlbetei- } \\
\text { ligung }\end{array}$ & $\begin{array}{l}\text { Gemeinde- } \\
\text { größe }\end{array}$ & $\begin{array}{l}\text { Gesell. } \\
\text { Heteroge- } \\
\text { nität }\end{array}$ & Finanzkraft & $\begin{array}{l}\text { Wettbewerb } \\
\text { im Partei- } \\
\text { ensystem }\end{array}$ & $\begin{array}{l}\text { Fraktionali- } \\
\text { sierungsgrad im } \\
\text { Parteiensystem }\end{array}$ \\
\hline Mean & 83,52 & 2926,58 & 3,17 & 641,39 & 25,42 & 2,39 \\
Std. & 5,60 & 2677,86 & 2,81 & 3400,54 & 14,76 & 0,43 \\
Abw & & & & & \\
Min & 68,55 & 257,00 & 0,14 & 31,52 & 0,06 & 1,00 \\
Median & 84,06 & 2301,50 & 2,42 & 632,11 & 24,08 & 2,36 \\
Max & 97,57 & 19501,00 & 19,42 & 2455,64 & 84,80 & 3,94 \\
\hline
\end{tabular}

Bei den Wahlen im Jahr 2009 fiel die Wahlbeteiligung allerdings wieder ab und 22 Gemeinden wiesen sehr niedrige Partizipationsraten auf.

Während die Anzahl der Gemeinden mit relativ niedriger Wahlbeteiligung vor allem in den 1990er-Jahren bis hin zur Wahl im Jahr 2009 deutlich wuchs, nahm die Anzahl der Gemeinden mit einer sehr hohen Wahlbeteiligung, von $90 \%$ und mehr (gemessen am 90. Percentil) im selben Zeitraum deutlich ab (siehe Abb. 3b). Im Jahr 1979 lag in 35 der 118 Gemeinden im Bundesland Salzburg die Wahlbeteiligung bei $90 \%$ oder mehr. In den Folgejahren sank die Anzahl dieser Gemeinden und ab dem Wahljahr 1999 fielen nur mehr sieben und dann acht Gemeinden in diese Kategorie. Das führte letztlich auch dazu, dass die durchschnittliche kommunale Wahlbeteiligung im 30-jährigen Beobachtungszeitraum, wenn auch nur leicht, aber eben dennoch gesunken ist.

Die Gemeindegrößen im Blick, zählten die hier untersuchten 118 Salzburg Gemeinden (ohne die Stadt Salzburg) im Zeitraum 1979 bis 2009 zwischen 257 (Tweng im Lungau) und 19.501 (Hallein im Tennengau) Einwohner und Einwohnerinnen. Die durchschnittliche Gemeindegröße lag (ohne die Stadt Salzburg) bei 2927 Einwohner und Einwohnerinnen. Nimmt man die Stadt Salzburg hinzu und stellt die durchschnittlichen Gemeindegrößen der österreichischen Bundesländer (ohne Wien) mit Stichtag 1.1.2010 gegenüber, zeigt sich, dass Salzburg, knapp gefolgt von Kärnten, die im Schnitt größten Gemeindestrukturen aufwies.

Tab. 1 fasst wichtige Kennwerte für das Kriterium (Wahlbeteiligung), die Testvariable (Gemeindegröße) und die Kontrollvariablen zusammen.

\section{Modell}

Erklärtes Ziel ist die Schätzung des Einflusses der Bevölkerungszahl und die Kontrolle weiterer sozio-ökonomischer und politisch-institutioneller Faktoren auf die Partizipationsraten bei Gemeindewahlen. Ein lineares Mixed-Model schafft dabei die Grundlagen, um im kontextuellen Setting die Effekte erklärender Variablen auf die ,Wahlbeteiligung bei Gemeindewahlen (VT)“ testen zu können. Zum Einsatz kommt ein Cross-Classified-Modell (siehe z. B. Garson 2013; Meyers und Beretvas 2006; Luo und Kwok 2009; Leckie 2013; Rasbash und Browne 2008; StataCorp 
Tab. 2 Wahlgang (1 bis 826): Cross-classified mit Gemeinde (118) und Wahljahr (7) (eigene Darstellung)

\begin{tabular}{lllllllll}
\hline Wahlgänge & & 1979 & 1984 & 1989 & 1994 & 1999 & 2004 & 2009 \\
\hline Gemeinden & Bruck & $\mathrm{X}$ & $\mathrm{X}$ & $\mathrm{X}$ & $\mathrm{X}$ & $\mathrm{X}$ & $\mathrm{X}$ & $\mathrm{X}$ \\
& Dienten & $\mathrm{X}$ & $\mathrm{X}$ & $\mathrm{X}$ & $\mathrm{X}$ & $\mathrm{X}$ & $\mathrm{X}$ & $\mathrm{X}$ \\
& Fusch & $\mathrm{X}$ & $\mathrm{X}$ & $\mathrm{X}$ & $\mathrm{X}$ & $\mathrm{X}$ & $\mathrm{X}$ & $\mathrm{X}$ \\
& Lend & $\mathrm{X}$ & $\mathrm{X}$ & $\mathrm{X}$ & $\mathrm{X}$ & $\mathrm{X}$ & $\mathrm{X}$ & $\mathrm{X}$ \\
& Rauris & $\mathrm{X}$ & $\mathrm{X}$ & $\mathrm{X}$ & $\mathrm{X}$ & $\mathrm{X}$ & $\mathrm{X}$ & $\mathrm{X}$ \\
& Taxenbach & $\mathrm{X}$ & $\mathrm{X}$ & $\mathrm{X}$ & $\mathrm{X}$ & $\mathrm{X}$ & $\mathrm{X}$ & $\mathrm{X}$ \\
& Zell am See & $\mathrm{X}$ & $\mathrm{X}$ & $\mathrm{X}$ & $\mathrm{X}$ & $\mathrm{X}$ & $\mathrm{X}$ & $\mathrm{X}$ \\
& Etc. $(N=118)$ & $\mathrm{X}$ & $\mathrm{X}$ & $\mathrm{X}$ & $\mathrm{X}$ & $\mathrm{X}$ & $\mathrm{X}$ & $\mathrm{X}$ \\
\hline
\end{tabular}

2013). ${ }^{7}$ Ausgangspunkt der Modellüberlegungen ist, dass die Einheiten der untersten Modellebene (Kommunalwahlen) nicht im hierarchischen Sinne in den oberen Ebenen ,genestet“ sind. Es liegen vielmehr Daten zu Wahlen (Level 1) in bestimmten Gemeinden (Level 2 - Kontext 1) zu bestimmten Zeitpunkten (Level 2 - Kontext 2) vor. Gemeinden und Wahljahre effektieren - „cross-classified“ und voneinander unabhängig - auf Level zwei (,two way cross classification“) und sind relevante Quellen der Kriterienvariation (Rasbash und Browne 2008, S. 303 f.).

Die Kreuztabelle (siehe Tab. 2 - Wahlgang [1 bis 826]: Cross-classified mit Gemeinde [118] und Wahljahr [7]) führt vor Augen, dass sowohl in Zeilen (für die Gemeinden, 118 Einheiten in Kontext 1) als auch in Spalten (für die Wahljahre, 7 Einheiten in Kontext 2) mehrfach Eintragungen für Wahlgänge (insgesamt 826) vorliegen. Würde ein „genestetes“ und damit hierarchisches Verhältnis vorliegen, wären die Beobachtungen für eine Gemeinde in immer nur einer Spalte (Wahljahr) eingetragen (Rasbash und Browne 2008, S. 303).

Die Cross-Classified-Struktur ermöglicht einerseits die Schätzung der fixen Parameter und andererseits die Kontrolle der kombinierten Random-Effects der Kontexte auf der zweiten Modellebene. Letztlich werden so zwei Modelle mit je einem Random-Intercept (einmal für die Gemeinden und einmal für die Wahljahre) gerechnet, schließlich kombiniert und gemeinsam in die Schätzung eingebracht. Effekte auf das Kriterium können dann allerdings nicht mehr als Wirkungen der einen oder anderen Modellebene oder des einen oder anderen Kontextes interpretiert werden, sondern müssen als verwoben und zwischen den Ebenen und Kontexten variierend verstanden werden (Fielding und Goldstein 2006, S. 22). Zunächst kann das zu Grunde liegende Modell in der Gleichung

$$
\mathrm{VT}_{\mathrm{ij}}=a+u_{i}+v_{j}+e_{i j}
$$

\footnotetext{
7 auch crossed-effects-model oder cross-classified linear mixed modeling - CCLMM, cross-classified multilevel measurement modeling - CCMMM, cross-classified random effects modeling - CCREM. Die Anwendung eines hierarchischen, gemischten Modells auf das vorliegende Datenset könnte zu verzerrten Schätzungen führen. Garson (2013) erklärt dazu: „Applying hierarchical linear mixed modeling to crossclassified data can seriously bias variance component estimates as well as bias the estimation of the standard errors of the regression coefficients ".
} 
Tab. 3 Übersicht der verwendeten Variablen

\begin{tabular}{lll}
\hline Abhängige Variable $(Y)$ & Kurzbezeichnung & Art \\
Wahlbeteiligung & VT & Orig \\
Unabhängige Variablen $(X i)$ & Kurzbezeichnung & Art \\
Gemeindegröße (Testmerkmal) & Sln_c & $\ln /$ zentriert \\
Gesell. Heterogenität/Tertiärisierungsgrad & TIln_c & $\ln /$ zentriert \\
Interaktion & IA_Sln_c_TIln_c_c & $\ln /$ zentriert \\
Finanzkraft & EEln_c & $\ln /$ zentriert \\
Wettbewerb im Parteiensystem & A_c & Zentriert \\
Fraktionalisierungsgrad im Parteiensystem & LaTa_c & Zentriert \\
\hline
\end{tabular}

formuliert werden, wobei a die Konstante, i die Gemeinde und j das Wahljahr klassifiziert. Das Kürzel $\mathrm{u}_{\mathrm{i}}$ gibt den Random-Intercept und damit zufälligen Effekt der Gemeinde und $\mathrm{v}_{\mathrm{j}}$ jenen des Wahljahres auf die Varianz der Wahlbeteiligung $\left(\mathrm{VT}_{\mathrm{ij}}\right)$ an. $\mathrm{VT}_{\mathrm{ij}}$, das Modellkriterium, ist die Wahlbeteiligung in einem bestimmten Wahlgang in einer bestimmten Gemeinde und ergibt sich aus der Cross-Classification zwischen einer Gemeinde i im Wahljahr j. Die Komponente $e_{i j}$ ist als Residuum auf der ersten Ebene und damit eines Wahlganges in einer Gemeinde i zu einem bestimmten Zeitpunkt $\mathrm{j}$ (Wahljahr) definiert (modelliert nach Rasbash und Browne 2008, S. 308 f.) Die in die Analysen einzubringenden fixen Faktoren sind in Tab. 3 dargestellt. Die Variablenkürzel lassen bereits erahnen, dass die ins Modell einzubringenden unabhängigen Variablen an ihrem Mittelwert, dem ,grand mean “, zentriert (Langer 2010, S. 57 ff; Braun et al. 2010, S. 31 ff.) und zum Teil logarithmiert wurden. Dieses Modell geht von der Annahme aus, dass die eingebrachten X-Merkmale in allen Kontexten fixe Effekte erzeugen und damit auf die gleiche Art und Weise Wirkung entfalten und keine kontextspezifische Variation gegeben ist. So variieren zwar die Konstanten auf der zweiten Modellebene (Random Effects), nicht aber die Steigungen der partiellen Parameter (Fixed Effects). Deren Kontextabhängigkeit wird im Mehrebenenmodell aber explizit erfasst.

Den Vorschlägen von Braun (2010), Langer (Langer 2010) und Baltes-Götz (2013) folgend, die dabei auf im Modellfeld prägende Verfahrensentwickler wie Snijders und Bosker (1999), Radenbusch und Bryk (2002) oder Hox (2010) verweisen, wird - hier in der Anwendung eines Cross-Classified-Modells - folgender „Fahrplan der Mehrebenanalyse“ eingehalten: In einem ersten Arbeitsschritt wird über die Schätzung eines Random-Intercept(s)-Only-Modells (RIO) die Kontextabhängigkeit der abhängigen Variable „Wahlbeteiligung bei Gemeindewahlen (VT)“ dargestellt (Langer 2010, S. 745 ff.). Dieses erste Modell, auch „Empty Model“ genannt, bietet Einblick in die Varianzaufteilung zwischen den Ebenen und Kontexten. Das Modell enthält nur die Konstante und kontextspezifische Varianzkomponenten (bezogen auf die untersuchten Gemeinden und Wahljahre), aber keine weiteren unabhängigen Variablen (Braun et al. 2010, S. 22 ff). So kann die Frage beantwortet werden, ob Varianzanteile der Wahlbeteiligung über die Kontexte der zweiten Ebene erklärt werden können (Langer 2010, S. 742). Wäre keine Variation auf der zweiten Ebene beobachtbar, könnte auf den Einsatz eines Mehrebenemodells verzichtet werden. Um den Mehrwert des kontextualisierten Settings besser einschätzen zu 
können, wird die Güte des geschätzten RIO-Modells jener des „Nullmodells“ mit fixer Konstante und ohne Kontexteffekte gegenübergestellt:

$$
\operatorname{RIO}(\text { Empty }): V T_{i j}=a+u_{i}+v_{j}+e_{i j} /(\text { Null }): \mathrm{VT}_{00}=a+e_{00}
$$

Lässt sich einerseits die Kontextabhängigkeit des Kriteriums nachweisen und andererseits die Modellgüte positiv entwickeln (RIO- vs Null-Modell), kann auf dieser Grundlage in einem zweiten Schritt ein Random-Intercept-Modell mit der Integration des erklärenden Testmerkmals (Population Size - Sln_c) gerechnet werden (RI_1: $\mathrm{RIO}+$ Sln_c):

$$
R I \_1: V T_{i j}=a+b_{1} * S \operatorname{Sn} \_c_{i j}+u_{i}+v_{j}+e_{i j}
$$

Im dritten Schritt wird die erste Kontrollvariable (Population Heterogeneity/ Tertiärisierungsgrad - TIln_c) und der Interaktionsterms, der sich aus dem multiplikativen Effekt der Gemeindegröße und Merkmalen der Tertiärisierung (TIln_c) ergibt, integriert (RI_2: RI_1 + TIln_c, IA_Sln_c_TIln_c_c):

$$
\begin{array}{r}
R I \_2: V T_{i j}=a+b_{1} * S \ln \_c i j+b_{2} * T I l n \_c_{i j}+ \\
b_{3} * I A \_S l n \_c \_T I l n \_c \_c_{i j}+u_{i}+v_{j}+e_{i j}
\end{array}
$$

Die Schritte eins bis drei der Analysefolge dienen primär der Verfahrensabsicherung und der Modellentwicklung und verschaffen einen ersten Einblick in die Prädiktoreneffekte. Der eigentliche Hypothesentest unter kontrollierten Bedingungen erfolgt in der vierten finalen Phase. Ergänzend zu den bereits gerechneten fixen Faktoren werden die restlichen Variablen der Alternativerklärungen integriert (final: RI_2 + EEln_c, A_c, LaTa_c):

$$
\begin{array}{r}
\text { (Final) }: V T_{i j}=a+b_{1} * T I l n \_c_{i j}+b_{2} * S \ln \_c_{i j}+ \\
b_{3} * I A \_S l n \_c \_T I l n \_c \_c_{i j}+b_{4} * E E l n \_c_{i j}+ \\
>b_{5} * A \_c_{i j}+b_{6} * L a T a \_c_{i j}+u_{i}+v_{j}+e_{i j}
\end{array}
$$

Die Umsetzung des Crossed-Effects-Modells ist mit dem Statistiksoftwarepaket STATA SE13 erfolgt. Im Folgenden wird die vorgezeichnete Analyse detailliert dargestellt.

Im ersten Schritt der Analyse liefert das RIO-Modell für die Konstante einen Wert in der Höhe von 83,52 (siehe Tab. 4 - Random Intercept(s)-Only-Modell [RIO/Empty]/Null-Modell). Dieser kann als mittlere Wahlbeteiligung über alle Wahlgänge (im Zeitablauf) und Gemeinden hinweg interpretiert werden (Braun et al. 2010, S. 29 ff). Als Varianzkomponenten gibt die oben angeführte Tabelle die Residualstandardabweichungen der Individual- $\left(\sigma_{e}\right)$ und der Kontextebene $\left(\sigma_{u} / \sigma_{v}\right)$ an. Da die Modelle in sich nicht ,genestet“ sind, ist der Einsatz eines LR-Tests in der Modellgegenüberstellung ausgeschlossen (Stata.com 2015). Der empfohlene Blick auf die AIC- und BIC-Werte (,smaller is better") zeigt aber klar die positive Modellentwicklung im RIO-Modell gegenüber dem Null-Modell an. Im Ergebnis kann festgehalten werden, dass die mittleren Wahlbeteiligungen zwischen den Kontext- 
Tab. 4 Random Intercept(s)Only-Modell (RIO/Empty)/ Null-Model

\begin{tabular}{|c|c|c|}
\hline & Null & RIO (Empty) \\
\hline \multicolumn{3}{|l|}{ Fixe Effekte } \\
\hline \multirow[t]{2}{*}{ Konstante (a) } & $83,520 * * *$ & $83,520 * * *$ \\
\hline & {$[0,195]$} & {$[0,921]$} \\
\hline \multicolumn{3}{|l|}{ Varianzkomponenten } \\
\hline Kontext $1 / \sigma_{\mathrm{u}}$ & - & $4,480 * * *$ \\
\hline Kontext $2 / \sigma_{\mathrm{v}}$ & - & $2,164 * *$ \\
\hline Individualebene/ $\sigma_{e}$ & $5,596 * * *$ & $2,714 * * *$ \\
\hline AIC & 5193,307 & 4380,198 \\
\hline BIC & 5202,740 & 4399,064 \\
\hline$N$ & 826 & 826 \\
\hline
\end{tabular}

einheiten signifikant variieren und somit die Modellwahl (Mehrebenenanalyse Cross Classified) und die Aufnahme der Random Intercepts unterstützt werden.

Nachdem die Varianz auf der Kontextebene festgehalten wurde, kann in einem zweiten Schritt die Testvariable in das Mehrebenenmodell integriert werden (siehe Tab. 5). Geht es um die Wirkung der Population Size wird sogleich der postulierte negative Effekt auf die Wahlbeteiligung deutlich.

Die Modellgüte im Blick, zeigt die Gegenüberstellung der AIC-Werte im Emptyund RI_2-Modell (4380,198: 4249,416) die positive Modellentwicklung an.

Erfolgt in einer dritten Analysephase die Hinzunahme der „Population Heterogeneity" (Tertiärisierunsgrad) als erste der Kontrollvariablen und die Modellintegration des Interaktionsterms, der sich aus der „Gemeindegröße“ und dem „Tertiärisierungsgrad“ ergibt (siehe Tab. 5: Mehrebenenmodelle, „RI_2“), wird zunächst deutlich, dass der erkannte negative Effekte der Population Size auf die Partizipation bei Gemeindewahlen stabil bleibt. Die Heterogenität der Gesellschaft entfaltet aber ebenso signifikant Einfluss auf das Kriterium, wobei der Effekt die erwartete negative Richtung annimmt. Mit dem Grad der Fraktionalisierung sinkt die Partizipationsrate. Zudem wird ein bedeutsamer Einfluss der eingebrachten Wechselwirkung angezeigt.

Die Analyse der Interaktion (siehe Abb. 4 unten) führt vor Augen, dass der Parameter wie erwartet wirkt. Der Koeffizient der Wahlbeteiligung sinkt, wenn der Interaktionsterm einen hohen Wert zeigt. Mit der Gemeindegröße verstärkt sich also der negative partizipatorische Effekt einer heterogenen lokalen gesellschaftlichen Struktur. Auch im Umkehrschluss kann argumentiert werden. In kleineren Gemeinden hat ein höherer gesellschaftlicher Fraktionalisierungsgrad eine geringere negative Wirkung auf die Partizipationsraten. Somit haben Fraktionalisierungseffekte in kleinen Gemeinden relativ geringe, in mittleren und größeren Gemeinden aber steigende Wirkungen auf das Ausmaß der Wahlbeteiligung. Die Richtung der Wechselwirkung im Blick, wird eine ordinale Interaktion angezeigt.

Dabei bleibt sowohl der Haupteffekt der „Gemeindegröße“ als auch jener des „Teritärisierungsgrades“ in Folge der Interaktion global interpretierbar (Bühner und Ziegler 2009, S. 415 ff.).

Geht es um die Güte des RI_2-Modells kann festgehalten werden, dass Akaikes Informationskriterium den Wert 4179,324 annimmt und damit unter dem AIC-Ni- 
Tab. 5 Mehrebenenmodelle - Cross-Classified

\begin{tabular}{|c|c|c|c|}
\hline & \multicolumn{3}{|l|}{ Modelle } \\
\hline & RI_1 & RI_2 & Final \\
\hline \multicolumn{4}{|l|}{ Fixe Effekte } \\
\hline \multirow[t]{2}{*}{ Gemeindegröße (Sln_c) } & $-4,478$ & $-3,129 * * *$ & $-3,174 * * *$ \\
\hline & {$[0,346]$} & {$[0,317]$} & {$[0,308]$} \\
\hline \multirow[t]{2}{*}{ Tertiärisierungsgrad (TIln_c) } & - & $-2,424 * * *$ & $-2,471 * * *$ \\
\hline & - & {$[0,304]$} & {$[0,311]$} \\
\hline \multirow[t]{2}{*}{ Interaktion SIn_c*TIln_c } & - & $-1,240 * * *$ & $-1,247 * * *$ \\
\hline & - & {$[0,158]$} & {$[0,159]$} \\
\hline \multirow[t]{2}{*}{ Finanzkraft (EEln_c) } & - & - & $-0,229$ \\
\hline & - & - & {$[0,474]$} \\
\hline \multirow[t]{2}{*}{ Wettbewerb im Parteiensystem (A_c) } & - & - & $-0,043 * * *$ \\
\hline & - & - & {$[0,011]$} \\
\hline \multirow[t]{2}{*}{ Fraktionalisierungsgrad im Parteiensystem (LaTa_c) } & - & - & 0,413 \\
\hline & - & - & {$[0,369]$} \\
\hline \multirow[t]{2}{*}{ Konstante (a) } & $83,520 * * *$ & $83,520 * * *$ & $83,520 * * *$ \\
\hline & {$[0,749]$} & {$[0,464]$} & {$[0,469]$} \\
\hline \multicolumn{4}{|l|}{ Varianzkomponenten } \\
\hline Kontext $1 / \sigma_{u}$ & $3,158 * * *$ & $2,498 * * *$ & $2,372 * * *$ \\
\hline Kontext $2 / \sigma_{\mathrm{v}}$ & $1,811^{*}$ & 1,039 & 1,072 \\
\hline Individualebene/ $\sigma_{e}$ & $2,613 * * *$ & $2,578 * * *$ & $2,544 * * *$ \\
\hline AIC & 4249,416 & 4179,324 & 4160,975 \\
\hline $\mathrm{BIC}$ & 4272,999 & 4212,341 & 4208,141 \\
\hline$N$ & 826 & 826 & 826 \\
\hline
\end{tabular}

SE in Klammern: *p $\leq 0,05, * * p \leq 0,01, * * * p \leq 0,001$

veau im RI_1-Modell liegt (4249,416). Die BIC-Werte zeigen das gleiche Bild und unterstreichen das Bild einer positiven Modellentwicklung.

Auffällig ist, dass in Folge der Integration der ersten Kontrollvariable und des Interaktionsterms die Varianzkomponente für das Kontextmerkmal „Wahljahre“ insignifikant wird. Ist demzufolge davon auszugehen, dass die resultierende Kontext2-Regressionskonstante nicht zufällig und über die beobachteten Jahre hinweg variiert? Es muss bedacht werden, dass für die Analysen Beobachtungen zu sieben Zeitpunkten (Wahljahre) herangezogen wurden. Die geringe Anzahl der Kontext-2Einheiten könnte die Insignifikanz der Varianzkomponente bedingen (Braun et al. 2010, S. 21). ${ }^{8}$ Vor diesem Hintergrund sollte die gegebene Insignifikanz der Varianzkomponente nicht vorschnell zum Schluss führen, dass hinsichtlich des betreffenden Merkmals eine systematische Kriterienvariation ausgeschlossen wäre. Der theore-

\footnotetext{
8 Braun et al. (2010, S. 21) geben mit Verweis auf Snijders (2003, p. 676) zu bedenken, ,,... dass weniger als 20 Fälle (i. e., Kontexteinheiten/Anm. d. Verf) nur eingeschränkte Rückschlüsse zulassen ..." und weniger als 10 Fälle Misstrauen hervorrufen sollten. Braun et al. (2010, S. 21) erklären aber auch darauf hin, ,,... dass bislang keine klare Konvention zur Fallzahlgröße vorherrscht, so dass alle Angaben nur als so genannte Daumenregeln zu verstehen sind“.
} 
Abb. 4 Interaktionseffekt I (a) und Interaktionseffekt II (b) a
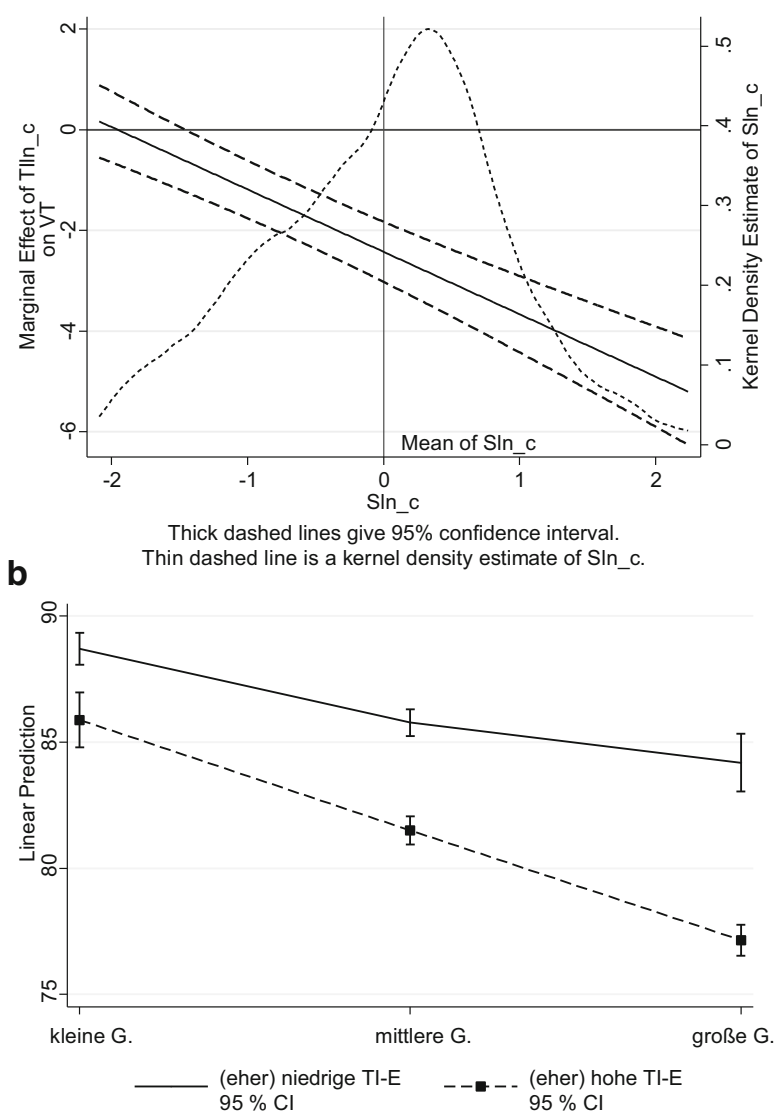

tisch als bedeutsam erachtete Faktor „Wahljahr“ wird deshalb weiterhin als relevante Modellkomponente für die Erklärung der Wahlbeteiligung bei Gemeindewahlen betrachtet und somit in die Analysen integriert.

In der vierten finalen Phase der Modellentwicklung werden eine weitere sozioökonomische Größe (Finanzkraft) und darüber hinaus politisch-institutionelle Faktoren (Abstand 1. und 2. Partei, Fraktionalisierungsgrad im Parteiensystem) in das Modell aufgenommen (siehe Tab. 5). So kann die Schätzung des Einflusses der Population Size unter Kontrolle von Störgrößen abgeschlossen werden. Im finalen Modell festigt sich der Eindruck, dass die Gemeindegröße entscheidend und indirekt-proportional Wirkung auf die Variation der abhängigen Variable entfaltet. Zudem gestalten Gemeinden, die einen hohen Heterogenitätsgrad aufweisen, ein Milieu aus, in dem die Wahlbeteiligung tendenziell niedrig ausfällt. Hinzu kommt die stabil moderierende Wirkung der „Gemeindegröße“ auf den Effekt des Fraktionalisierunsgrads der lokalen Gesellschaft. Der ohnehin geringe - negative - Effekt der ökonomischen Rahmenbedingungen bleibt insignifikant. Eine günstige materielle Ressourcenausstattung wirkt sich demnach nicht wie erwartet positiv, sondern so gut wie nicht auf die Partizipationsrate bei Gemeindewahlen aus. Geht es um die 
Tab. 6 Einflussfaktoren auf die Partizipationsraten bei Gemeindewahlen

\begin{tabular}{llll}
\hline & Merkmalsausprägung & $\begin{array}{l}\text { Y-Variable Wahlbe- } \\
\text { teiligung } \\
\text { Erwartung }\end{array}$ & $\begin{array}{l}\text { Y-Variable Wahl- } \\
\text { beteiligung } \\
\text { Hypothesentest }\end{array}$ \\
\hline $\begin{array}{l}\text { Testvariable } \\
\text { Gemeindegröße (Sln_c) }\end{array}$ & Hoch & Niedrig \\
$\begin{array}{l}\text { Kontrollvariablen } \\
\text { Sozio-ökonomische Faktoren }\end{array}$ & Hoch & Niedrig \\
$\begin{array}{l}\text { Gesell. Heterogenität/ } \\
\text { Tertiärisierungsgrad (TIln_c) }\end{array}$ & Hoch & Niedrig \\
$\begin{array}{l}\text { Interaktionseffekt } \\
\text { (IA_Sln_c \& TIln_c_c) }\end{array}$ & Niedrig & Niedrig \\
$\begin{array}{l}\text { Finanzkraft (EEln_c) } \\
\begin{array}{l}\text { Politisch-institutionelle Faktoren } \\
\text { Wettbewerb im Parteiensystem }\end{array}\end{array}$ & Hoch & Hoch & Niedrig \\
$\begin{array}{l}\text { A_c) } \\
\begin{array}{l}\text { Fraktionalisierungsgrad (La- } \\
\text { Ta_c) }\end{array}\end{array}$ & Hoch & Niedrig & Nicht signifikant \\
\hline
\end{tabular}

politisch-institutionellen Faktoren wird klar, dass ein enges Rennen zwischen den Parteien einen wichtigen Beitrag zur Wähler- und Wählerinnenmobilisierung leistet (Abstand zwischen 1. und 2. Partei). Unerheblich hingegen ist, ob die Palette an politischen Bewerbern ausgeprägt ist oder nicht. Die effektive Anzahl der Parteien hat keine relevante Einflusskraft auf die lokale Wahlbeteiligung.

Die Güte des Modells vor Augen zeigt sich, dass die Integration von Kovariaten, die über die Faktoren „Gemeindegröße“ und „Tertiärisierungsgrad“ sowie deren Interaktion hinausgehen, nicht mehr zu einer nennenswerten Modellverbesserung führt (AIC RI_2: 4179,324/AIC Final: 4160,975). Unterstrichen wird die Erkenntnis durch den BIC-Wert im finalem Modell $(4208,14)$, der nur minimal unter dem RI2_Modell liegt (4212,341). Dieses Ergebnis führt die durchschlagende Wirkung lediglich zweier Prädiktoren vor Augen. Auf das Kriterium wirken vor allem die Haupteffekte der Population Size und der Population Heterogeneity sowie deren Wechselwirkung. Tab. 6 resümiert die Ergebnisse gegenübergestellt mit den eingangs formulierten Erwartungen.

\section{Schlusswort}

Erklärtes Ziel war der Test von Erklärungsansätzen für variierende Wahlbeteiligungen bei Gemeindewahlen. Ausgehend vom bereits vielfach beobachteten negativen Zusammenhang zwischen Population Size und Partizipationsrate, der durch neue Forschung in Frage gestellt wurde, wurde das Argument rund um die Gemeindegröße mit ihrem Effekt auf die Wahlbeteiligung bei Gemeindewahlen entwickelt. Der Hypothesentest wurde sodann mit Berücksichtigung weiterer sozio-ökonomischer sowie politisch-institutioneller Alternativerklärungen vorbereitet. Das dem Test zugrunde liegende umfangreiche Datenmaterial - 118 Gemeinden im Bundesland Salzburg bei sieben Wahlen von 1979 bis 2009/826 Wahlgänge - erlaubte die Kontrolle 
der Einflüsse weiterer politisch-institutioneller Merkmaler auf Partizipationsraten bei Wahlen, wie das Wahlsystem, die Gleichzeitigkeit von Wahlen (Concurrent Elections) oder einer bestimmten Tradition im Wahlverhalten (Previous Turnout). Somit konnten wir durch die Art der Fallauswahl diese wichtigen latenten Variablen kontrollieren und den Einfluss sozio-kultureller Veränderungsprozesse und eventuell gegebener „Traditionen im lokalen Wahlverhalten“ berücksichtigen. Allerdings kann mit den unserer Arbeit zugrunde liegenden Aggregatdaten keine Aussage für die Individualebene getätigt werden, dennoch lassen sich eindeutige Rückschlüsse auf die Ursachen variierender Wahlbeteiligungen ziehen.

Zunächst zeigte sich, dass vor allem sozio-ökonomische Faktoren und hier im hohen Ausmaß die Gemeindegröße und der Grad gesellschaftlicher Fraktionalisierung sowie deren Wechselwirkung die Wahlbeteiligung bei Gemeindewahlen beeinflussen. Die Hypothesenprüfung finalisierend kann festgehalten werden, dass hinsichtlich des Testfaktors „Population Size“ kontrolliert und signifikant negative Effekte auf die Variation der Wahlbeteiligung bei Gemeindewahlen nachgewiesen werden konnten. In kleineren Gemeinden liegen die Partizipationsraten höher als in größeren Gemeinden. Die erwarteten Zusammenhänge wurden wiederholt und auch in der hier angestellten Untersuchung beobachtet. Das höhere relative Gewicht der einzelnen Stimme und die leichte Überschaubarkeit des unmittelbaren Lebensumfeldes, sei es hinsichtlich der politischen Akteure oder sei es hinsichtlich der politischen Vorgänge, wirken sich günstig auf die Beteiligung bei Gemeindewahlen aus. Die „Gemeindegröße“ moderiert zudem den Effekt des Faktors „Population Heterogeneity “ in der Art, dass sich die negative Wirkung eines hohen Niveaus gesellschaftlicher Fraktionalisierung auf die Partizipationsrate in kleineren Gemeinden weniger stark auswirkt.

Interessanterweise bleibt jedoch die Wirkung politisch-institutioneller Größen generell gering, wenngleich nicht vergessen werden darf, dass das Vorhandensein eines Parteienwettbewerbs sich positiv auf die Partizipationsraten bei Gemeindewahlen auswirkt.

Wenn wir den negativen Effekt gesellschaftlicher Diversität auf die Partizipationsrate bei Gemeindewahlen betrachten, gilt es zu bedenken, dass eher heterogen strukturierte Gemeinden häufig von einer starken Zuwanderung geprägt sind. Davon betroffen sind vor allem die regionalen und überregionalen Zentren und ihre Umlandgemeinden. Das führt zur Überlegung, dass interlokale Wanderungen in die regionalen und überregionalen Arbeitsmarktzentren und damit weg vom ruralen hin zum urbanen Raum das kommunale Partizipationsverhalten beeinflussen. Diese Mobilität führt einerseits dazu, dass sich in Abwanderungsgemeinden das Potential für lokale politische Gestaltung verringert. Andererseits entfaltet sich auch in der Zuwanderungsgemeinde wenig bis gar kein Interesse an einer lokalen politischen Beteiligung, weil für Zugewanderte und damit zunächst ortsfremde Personen die Kosten der Partizipation hoch sind.

Geht es um die Diskussion der Pros und Kontras in puncto Strukturreformen auf lokaler Ebene, so liefern die Ergebnisse unserer Arbeit einen klaren Hinweis darauf, dass eine Zusammenlegung von Gemeinden Kosten in Gestalt einer sinkenden Wahlbeteiligung verursacht.

Rückgeführt auf die eingangs erwähnte demokratietheoretische Diskussion, kann eine niedrige Wahlbeteiligung in stark diversifizierten lokalen Gesellschaften entste- 
hen, weil bestimmte gesellschaftliche Fraktionen nicht (mehr) politisch partizipieren. Unsere Arbeit impliziert somit, dass weitere Forschung diese Zusammenhänge aufgreifen und der Frage nachgehen müsste, ob schon heute, vornehmlich im eher urbanen Raum, nur (mehr) bestimmte Bevölkerungsgruppen von ihrem politischen Beteiligungsrecht, insbesondere bei Gemeindewahlen, Gebrauch machen und benachteiligte gesellschaftliche Gruppen großteils ausgeschlossen sind.

Open access funding provided by Paris Lodron University of Salzburg.

Open Access Dieser Artikel wird unter der Creative Commons Namensnennung 4.0 International Lizenz (http://creativecommons.org/licenses/by/4.0/deed.de) veröffentlicht, welche die Nutzung, Vervielfältigung, Bearbeitung, Verbreitung und Wiedergabe in jeglichem Medium und Format erlaubt, sofern Sie den/die ursprünglichen Autor(en) und die Quelle ordnungsgemäß nennen, einen Link zur Creative Commons Lizenz beifügen und angeben, ob Änderungen vorgenommen wurden.

\section{Literatur}

Aldrich, John H. 1997. When is it rational to vote? In Perspectives on public choice, Hrsg. Dennis C. Mueller, 373-390. Cambrige: Cambridge University Press.

Anderwald, Karl. 2008. Kommunalpolitik und Kommunalverwaltung. In Handbuch kommunales Management: Rahmenbedingungen, Aufgabenfelder, Chancen und Herausforderungen, Hrsg. Dietmar Brodel, 23-32. Wien: Lexis Nexis, ARD Orac (Orac Wirtschaftspraxis).

Baethge, Martin. 2000. Abschied vom Industrialismus: Konturen einer neuen gesellschaftlichen Ordnung der Arbeit. SOFI-Mitteilungen 28:87-101.

Baltes-Götz, Bernhard. 2013. Analyse von hierarchischen linearen Modellen mit der SPSS-Prozedur MIXED. Trier: Universität Trier. Zentrum für Informations-, Median-, und Kommunikationstechnologie (ZIMK). https://www.uni-trier.de/fileadmin/urt/doku/hlm/hlm.pdf. Zugegriffen: 12. Mai 2015.

Bhatti, Yosef, und Kasper Hansen. 2010. Kommunalreformens betydning for den kommunale valgdeltagelse. Tidsskriftet Politik 13(3):6-16.

Blais, André, und Agnieszka Dobrzynska. 1998. Turnout in electoral democracies. European Journal of Political Research 33(2):239-261.

Braun, Daniela, Nicole Seher, Markus Tausendpfund, und Ansgar Wilsong. 2010. Einstellungen gegenüber Immigranten und die Zustimmung zur Europäischen Union. Eine Mehrebenenanalyse. Arbeitspapiere/Working Papers 136. Mannheim: Mannheimer Zentrum für Europäische Sozialforschung. http:// www.mzes.uni-mannheim.de/publications/wp/wp-136.pdf. Zugegriffen: 12. Mai 2015.

Bühner, Markus, und Matthias Ziegler. 2009. Statistik für Psychologen und Sozialwissenschaftler. München: Addison-Wesley.

Coffe, Hilde, und Benny Geys. 2006. Community heterogeneity: a burden for the creation of social capital? Social Science Quarterly 87(5):1053-1072.

Dahl, Robert A., und Edward R. Tufte. 1973. Size and democracy. Stanford: Stanford University Press.

Dalton, Russell J. 1996. The History of Party Systems. In Citizen Politics: Public Opinion and Political Parties in Advanced Western Democracies, Hrsg. Russell J. Dalton, 149-164. Chatham, NJ: Chatham House.

Denters, Bas, Michael Goldsmith, Andreas Ladner, Poul Erik Mouritzen, und Lawrence E. Rose. 2014. Size and local democracy. Cheltenham: Edward Elgar Publishing.

Dolezal, Martin, und Klaus Poier. 2012. Rechtliche Hürden, sozioökonomischer Kontext oder Struktur des Parteienwettbewerbs? Die Anwendung direktdemokratischer Verfahren in Österreichs Gemeinden. Arbeitspapier präsentiert auf der zweiten Wissenschaftskonferenz der ÖFG. Wien: ÖFG Arbeitsgruppe Zukunft der Demokratie. http://www.oefg.at/wp-content/uploads/2014/08/dolezal_poier.pdf. Zugegriffen: 08. Mai 2015.

Elkmann, Reinhard, Nora Lauterbach, und Stephan Wind. 1994. Tertiärisierung regionaler Wirtschaftsstrukturen: eine empirische Analyse kreisfreier Städte und Landkreise in Hessen, Rheinland-Pfalz und dem Saarland. Arbeitspapiere des Instituts für Statistik und Ökonometrie 6. Mainz: STATOEK. http://econstor.eu/bitstream/10419/49969/1/257644997.pdf. Zugegriffen: 06. Mai 2015.

Feld, Scott, und Bernhard Grofman. 2007. The Laakso-Taagepera-Index in a mean and variance framework. Journal of Theoretical Politics 19(1):101-106. 
Fielding, Anthony, und Harvey Goldstein. 2006. Cross-classified and multiple membership structures in multilevel models: an introduction and review. Research Report, Bd. 791. Birmingham: University of Birmingham.

Filipp, Gernot, und Peter Kurz. 2014. Bevölkerung Land Salzburg. Stand \& Entwicklung 2014. Themenschwerpunkt: Geburten. Salzburg: Amt der Salzburger Landesregierung.

Freitag, Markus. 1996. Wahlbeteiligung in westlichen Demokratien. Eine Analyse zur Erklärung von Niveauunterschieden. Swiss Political Science Review 2(4):1-35.

Garson, G. David. 2013. Fundamentals of hierarchical linear and multilevel modeling. In Hierarchical linear modeling. Guide and applications, Hrsg. David Garson, 3-26. Thousand Oaks: SAGE.

Geys, Benny. 2006. Explaining voter turnout: a review of aggregate-level research. Electoral Studies 25(4):637-663.

Hansen Welling, Sune. 2013. Polity size and local political trust: a quasi-experiment using municipal mergers in Denmark. Scandinavian Political Studies 36(1):43-66.

Heinisch, Reinhard, Thomas Lehner, Armin Mühlböck, und Christian Schimpf. 2016. Subtraction by addition? The effect of municipality amalgamations on voter turnout in local elections. Paper präsentiert auf der MPSA General Conference. Chicago: MPSA.

Hicks, Bruce M. 2006. Are marginalized communities disenfranchised? Voter turnout and representation in post-merger Toronto. IRPP Working Paper Series, Bd. 2006-03. Montreal: Institute for Research on Public Policy.

Horiuchi, Yusaku, Jun Saito, und Kyohei Yamada. 2015. Removing boundaries, losing connections: electoral consequences of local government reform in Japan. Journal of East Asian Studies 15(1):99-125.

Hox, Joop J. 2010. Multilevel analysis. Techniques and applications, 2. Aufl., New York: Routledge.

Justel, Manuel. 1995. Electoral abstention in Spain: characteristics and factors. In Electoral abstention in Europe, Hrsg. Joan Font, Rosa Viros, 43-84. Barcelona: ICPS.

Kaniovski, Serguei, und Dennis C. Mueller. 2006. Community size, heterogeneity and voter turnouts. Public Choice 129:399-415. doi:10.1007/s11127-006-9063-7.

Kenneth, Newton. 1982. Is small really so beautiful? Is big really so ugly? Size, effectiveness, and democracy in local government. Political Studies 30(2):190-206.

Kohler, Ulrich. 2006. Die soziale Ungleichheit der Wahlabstinenz in Europa. In Europas Osterweiterung: Das Ende der Vertiefung? Hrsg. Jens Alber und Wolfgang Merkel, 159-182. Berlin: edition sigma.

Kornelius, Bernhard, und Dieter Roth. 2004. Politische Partizipation in Deutschland. Ergebnisse einer repräsentativen Umfrage. Gütersloh: Bertelsmann.

Kushner, Joseph, David Siegel, und Hannah Stanwick. 1997. Ontario municipal elections: voting trends and determinants of electoral success in a Canadian province. Canadian Journal of Political Science 30(3):539-553. doi:10.1017/S0008423900016000.

La Ferrara, Eliana. 2002. Inequality and group participation: theory and evidence from rural Tanzania. Journal of Public Economics 85:235-273.

Ladner, Andreas. 2002. Size and direct democracy at the local level: the case of Switzerland. Environmental Planning 20(6):813-828.

Ladner, Markus. 2009. What explains electoral turnout in Swiss municipalities? Arbeitspapier präsentiert auf der fünften ECPR General Conference. Potsdam: ECPR. http://my.unil.ch/serval/document/BIB_ 3ACEFE6604FB.pdf. Zugegriffen: 08. Mai 2015.

Langer, Wolfgang. 2010. Mehrebenenanalyse mit Querschnittsdaten. In Handbuch der sozialwissenschaftlichen Datenanalyse, 1. Aufl., Hrsg. Christof Wolf, Henning Best, 741-774. Wiesbaden: VS.

Leckie, George. 2013. Cross-classified multilevel models using STATA: how important are schools and neighborhoods for children's educational attainment? In Hierarchical linear modeling. Guide and applications, Hrsg. G. David Garson, 311-332. Thousand Oaks: SAGE.

Lijphart, Arend. 1997. Unequal participation: democracy's unresolved dilemma. American Political Science Review 19:1-14.

Lindert, Peter H. 1996. What limits social spending? Explorations in Economic History 33:1-34.

Luo, Wen, und Oi Kwok. 2009. The impacts of ignoring a crossed factor in analyzing cross-classified data. Multivariate Behavioral Research 44(2):182-212.

Marxer, Wilfried. 2004. Demokratie? Erscheinungsformen einer Idee. Schriftliche Fassung des Vortrages am Liechtenstein-Institut vom 19. Oktober 2004 in der Vorlesungsreihe „Herausforderung Demokratie “. Beiträge Liechtenstein-Institut, Bd. 24/2004

Merkel, Wolfgang, und Alexander Petring. 2011. Partizipation und Inklusion, in: Demokratie in Deutschland 2011 - Ein Report der Friedrich-Ebert-Stiftung. http://www.wzb.eu/sites/default/files/zkd/dsl/ partizipation_und_inklusion.pdf. Zugegriffen: 15. Januar 2013.

Meyers, Jason L., und Natasha S. Beretvas. 2006. The impact of inappropriate modeling of cross-classified data structures. Multivariate Behavioral Research 41(4):473-497. 
Miguel, Edward, und Mary K. Gerty. 2005. Ethnic diversity, social sanctions and public goods in Kenya. Journal of Public Economics 89:2325-2368.

Morlan, Robert L. 1984. Municipal vs. national election voter turnout: Europe and the United States. Political Science Quarterly 99(3):457-470.

Mouritzen, und Erik Poul. 1989. City size and citizens' satisfaction: two competing theories revisited. European Journal of Political Research 17(6):661-688.

Mühlböck, Armin. 2010. Die Zukunft der Gemeinden am Land. In Salzburger Jahrbuch für Politik 2010, Bd. 180, Hrsg. Herbert Dachs, Roland Floimair, 129-143. Wien: Böhlau.

Mühlböck, Armin. 2013. Die Gemeinden. In Übergänge und Veränderungen: Salzburg vom Ende der 1980er-Jahre bis ins neue Jahrtausend, Hrsg. Herbert Dachs, Christian Dirninger, \& Roland Floimair, 221-272. Wien: Böhlau.

Mühlböck, Armin, und Christian Dirninger. 2010. Leere Dörfer, volle Städte. Der demografische Wandel im ländlichen Raum Salzburgs. In Salzburger Jahrbuch für Politik 2010, Hrsg. Herbert Dachs, Roland Floimair, 45-62. Wien: Böhlau.

Powell, Bingham G. 1982. Contemporary democracies: participation, stability, and violence. Cambridge: Harvard University Press.

Rallings, Colin, Michael Temple, und Michael Trasher. 1994. Community identity and participation in local democracy. Research report. London: The Commission for Local Democracy.

Rasbash, Jon, und William Browne. 2008. Non-hierarchical multilevel models. In Handbook of multilevel analysis, Hrsg. Jan de Leeuw, Erik Meijer, 303-336. New York: Springer.

Raudenbush, Stephen W., und Anthony S. Bryk. 2002. Hierarchical linear models. Applications and data analysis methods, 2. Aufl., Thousand Oaks: SAGE.

Roth, Roland. 2009. Handlungsoptionen zur Vitalisierung der Demokratie. Expertise für die Bertelsmann Stiftung. http://www.b-b-e.de/fileadmin/inhalte/aktuelles/2009/09/n120_bertelsmann_vitalisierung. pdf. Zugegriffen: 15. November 2015.

Schäfer, Armin. 2010. Die Folgen sozialer Ungleichheit für die Demokratie in Westeuropa. Zeitschrift für Vergleichende Politikwissenschaft 4 (1): 131-156.

Schipfer, Rudolf Karl. 2005. Der Wandel der Bevölkerungsstruktur in Österreich: Auswirkungen auf Regionen und Kommunen. Wien: Österreichisches Institut für Familienforschung (ÖIF).

Schmidt, Manfred G. 2000. Demokratietheorien. Eine Einführung. Opladen: Leske + Budrich.

Shimizu, Kay. 2012. Electoral consequences of municipal mergers. Journal of East Asian Studies 12(3):381-408.

Shinn, Paul. 1999. Citizen participation and municipal government in three Oklahoma communities. Oklahoma Politics 8:17-42.

Snijders, Tom A.B., und Roel Bosker. 1999. Multilevel analysis: an introduction to basic and advanced multilevel modeling. London: SAGE.

Stata.com. 2015. xtmixed - multilevel mixed-effects linear regression. http://www.stata.com/bookstore/ pdf/xt_xtmixed.pdf. Zugegriffen: 12. Mai 2015.

StataCorp. 2013. Stata: release 13. statistical software. College Station: StataCorp LP.

Steininger, Barbara. 2006. Gemeinden. In Politik in Österreich: Das Handbuch, Hrsg. Herbert Dachs, Peter Gerlich, Herbert Gottweis, Helmut Kramer, Volkmar Lauber, Wolfgang C. Müller, \& Emmerich Tálos, 990-1007. Wien: Manz.

Topf, Richard. 1995. Electoral Participation. In Citizen and the state, Hrsg. Hans-Dieter Klingemann und Dieter Fuchs, 28-51. Oxford, UK: Oxford University Press.

Verba, Sidney. 2003. Would the dream of political equality turn out to be a nightmare? Perspectives in Politics 1:663-679.

Verba, Sidney, Norman H. Nie, und Kim Jae-on. 1979. Participation and political equality: a seven-nation comparison. Cambridge: Cambridge University Press.

Vetter, Angelika. 2002. Lokale Politik als Ressource der Demokratie in Europa? Lokale Autonomie, lokale Strukturen und die Einstellungen der Bürger zur lokalen Politik. Wiesbaden: VS.

Vetter, Angelika. 2008. Institutionen und lokale Wahlen: Wo bleiben die Wähler? In Erfolgsbedingungen lokaler Bürgerbeteiligung, 1. Aufl., Bd. 16, Hrsg. Angelika Vetter, 49-72. Wiesbaden: VS.

Zimmer, Troy A. 1976. Urbanization, social diversity, voter turnout, and political competition in US elections: Analysis of congressional districts for 1972. Social Science Quarterly 56(4):689-697.

Prof. Reinhard Heinisch Professor of Austrian Politics in European Perspective

Armin Mühlböck Senior Scientist 